Portland State University

PDXScholar

Summer 1-1-2012

\title{
Material Characterization of Zinc Oxide in Bulk and Nanowire Form at Terahertz Frequencies
}

Forest Emerson Kernan

Portland State University

Follow this and additional works at: https://pdxscholar.library.pdx.edu/open_access_etds

Part of the Electrical and Electronics Commons, and the Other Electrical and Computer Engineering Commons

Let us know how access to this document benefits you.

\section{Recommended Citation}

Kernan, Forest Emerson, "Material Characterization of Zinc Oxide in Bulk and Nanowire Form at Terahertz Frequencies" (2012). Dissertations and Theses. Paper 510.

https://doi.org/10.15760/etd.510

This Thesis is brought to you for free and open access. It has been accepted for inclusion in Dissertations and Theses by an authorized administrator of PDXScholar. Please contact us if we can make this document more accessible: pdxscholar@pdx.edu. 
Material Characterization of Zinc Oxide in Bulk and Nanowire Form at Terahertz Frequencies

\author{
by
}

Forest Emerson Kernan

A thesis submitted in partial fulfillment of the requirements for the degree of

\author{
Master of Science \\ in \\ Electrical and Computer Engineering
}

Thesis Committee:

Branimir Pejcinovic, Chair

Lisa M. Zurk

Rolf Könenkamp

Portland State University

2012 


\section{Abstract}

Many new applications are being proposed and developed for use in the terahertz $(\mathrm{THz})$ frequency region. Similarly, many new materials are being characterized for possible use in this area. Nanostructured forms are of particular interest since they may yield desirable properties, but they remain especially challenging to characterize. This work focuses on the characterization of zinc oxide $(\mathrm{ZnO})$ in bulk and nanowire form.

A method for characterizing nanostructures at $\mathrm{THz}$ by use of a parallel-plate waveguide (PPWG) is presented. This method is novel in that it is simple, both in theory and practice, and does not require the use of complex measurement techniques such as differential and double modulated terahertz time-domain spectroscopy (THz-TDS). To enable easy evaluation of the quality of the result the maximum deviation in the material response measurement is presented.

The dielectric properties of bulk and nanowire $\mathrm{ZnO}$ as determined by THz-TDS measurements are reported, and the electrical conductivity extracted from both are presented for comparison. Experimental results are compared to the well established pseudo-harmonic phonon dielectric model. Shortcomings in the pseudo-harmonic phonon model are resolved when coupled with a modified Drude model.

This work will enable the determination of $\mathrm{THz}$ material properties from nanoscale and very-thin film materials with better reliability and practicality than what has been possible until now. 


\section{Acknowledgements}

I would like to thank the Oregon Nanoscience and Microtechnologies Institute (ONAMI) for funding this research and I would like to thank the Center for Electron Microscopy and Nanofabrication (CEMN) for the use of their equipment and expertise in capturing SEM images. I would like to thank Athavan Nadarajah for

providing the $\mathrm{ZnO}$ nanowires, without whom this research would not have been possible. I would like to thank Alex Higgins who was instrumental in designing and manufacturing the PPWG, and for his feedback and support throughout this process. I would like to thank my advisor, Branimir Pejcinovic, who made this research possible and for his guidance and insight. And finally, I would like to thank my mother and father for pushing me forward in the right direction. 


\section{Contents}

Abstract $\quad$ i

$\begin{array}{ll}\text { Acknowledgements } & \text { ii }\end{array}$

List of Tables $\quad$ v

List of Figures $\quad$ vi

1 Introduction 1

1.1 Thesis Statement . . . . . . . . . . . . . . . 1

1.2 Thesis Outline . . . . . . . . . . . . . . . . . . 1

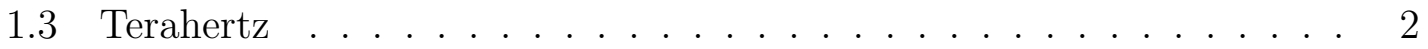

$1.4 \mathrm{ZnO} \ldots \ldots \ldots \ldots \ldots \ldots$

2 Terahertz Time-Domain Spectroscopy 6

2.1 THz-TDS System . . . . . . . . . . . . . . . . 6

3 Parallel-Plate Waveguides $\quad 8$

3.1 PPWG Application . . . . . . . . . . . . . . . . . . . 8

3.1.1 Transverse Magnetic Mode . . . . . . . . . . . . . . . . . . . . 9

3.1 .2 Construction ....................... 11

3.1.3 Experimental Setup . . . . . . . . . . . . . 13 
$\begin{array}{llr}4 \text { Zinc Oxide } & 15\end{array}$

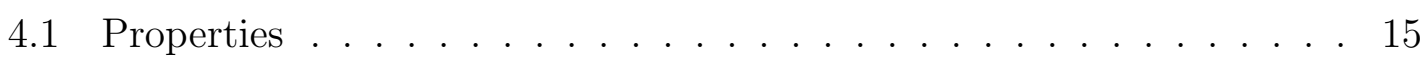

4.2 Nanowire Growth . . . . . . . . . . . . . . . . 16

5 Material Parameter Extraction $\quad 22$

5.1 Inverse Model . . . . . . . . . . . . . . . . . . . . . 22

5.1 .1 Free space .................... 22

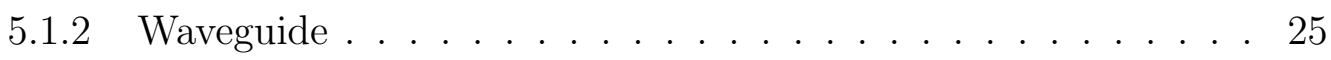

5.2 Effective Medium Model . . . . . . . . . . . . . . 26

5.3 Measurement Uncertainty . . . . . . . . . . . . . . . 27

6 Dielectric Theory 32

6.1 Dielectric Model . . . . . . . . . . . . . . . . . . . . 32

6.2 Complex Conductivity . . . . . . . . . . . . . . 37

7 Experimental Results $\quad 40$

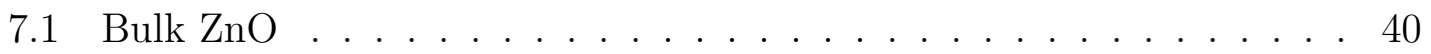

$7.2 \mathrm{ZnO}$ Nanowires ...................... 50

7.3 Material Comparison ................... 57

8 Conclusion $\quad 60$

9 Future Work $\quad 62$

$\begin{array}{ll}\text { References } & 63\end{array}$

$\begin{array}{ll}\text { Appendix } & 68\end{array}$

$\begin{array}{ll}\text { A Measurement Deviation } & 68\end{array}$ 


\section{List of Tables}

7.1 Model Parameters for Bulk Crystalline $\mathrm{ZnO}$. . . . . . . . . . . . . . 49

7.2 Model Parameters for $\mathrm{ZnO}$ Nanowires . . . . . . . . . . . . . . 57

7.3 Modeled Material Parameter Comparison . . . . . . . . . . . . . . 58 


\section{List of Figures}

3.1 ZnO nanowire deposition on gold plated PPWG. . . . . . . . . . . . . 12

3.2 Gold plated PPWG in experimental setup. . . . . . . . . . . . . . . . 12

3.3 Diagram of the PPWG experimental setup. . . . . . . . . . . . . 14

3.4 Using the THz-TDS system to detect misalignment. . . . . . . . . . . 14

4.1 SEM image of the first attempt at $\mathrm{ZnO}$ nanowire growth on aluminum. 18

4.2 SEM image of the second attempt at $\mathrm{ZnO}$ nanowire growth on anodized aluminum. . . . . . . . . . . . . . . . . 18

4.3 SEM image of the third attempt at $\mathrm{ZnO}$ nanowire growth on top of a thin film layer on aluminum. . . . . . . . . . . . . . . . . . 19

4.4 Aluminum deformations interspersed in $\mathrm{ZnO}$ nanowires. . . . . . . . . 19

4.5 SEM image of the successful $\mathrm{ZnO}$ nanowire growth on gold plated

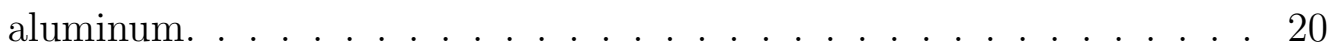

4.6 SEM image of $\mathrm{ZnO}$ nanowires grown on gold sputtered silicon. . . . . 20

4.7 Height measurement from an SEM image of $\mathrm{ZnO}$ nanowires grown on gold sputtered silicon. . . . . . . . . . . . . . . . . . . 21

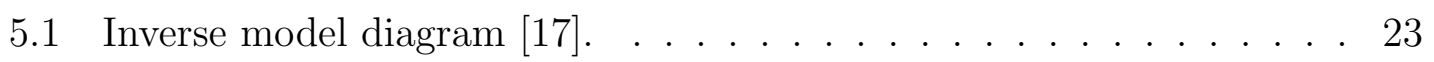


5.2 Frequency span over which reliable results were obtained for bulk $\mathrm{ZnO}$. Interference from the fixture used to hold the bulk $\mathrm{ZnO}$ wafer may be a contributing factor at the lower bound, as can be seen in Figures 7.6 and $7.7 \ldots \ldots \ldots \ldots$

5.3 Frequency span over which reliable results can be obtained for $\mathrm{ZnO}$ nanowires in a PPWG. . . . . . . . . . . . . . . . . . . . 29

5.4 Maximum deviation in the magnitude response of Bulk $\mathrm{ZnO}$. . . . . 30

5.5 Maximum deviation in the phase response of Bulk $\mathrm{ZnO} . \quad \ldots . . . .30$

5.6 Maximum deviation in the magnitude response of $\mathrm{ZnO}$ nanowires. . . 31

5.7 Maximum deviation in the phase response of $\mathrm{ZnO}$ nanowires. . . . . . 31

7.1 Experimentally measured time domain response. . . . . . . . . . . . 42

7.2 Experimentally measured frequency domain response. . . . . . . . . . 42

7.3 The total variation method as applied to the $\mathrm{ZnO}$ wafer to determine actual vs. nominal thickness. . . . . . . . . . . . . . . . 43

7.4 Experimentally determined angle of incidence . . . . . . . . . . . 43

7.5 Calculated Refractive index using assumed thickness of $0.5 \mathrm{~mm}$ and normal incident angle vs. final estimate of $512.8 \mu \mathrm{m}$ at a $4.53^{\circ}$ incidence. 44

7.6 The extracted real dielectric response from bulk crystalline $\mathrm{ZnO}$ as compared to the pseudo-harmonic phonon model (dashed, eq. (6.3)), and the modified Drude coupled plasmon-phonon model (solid, eq.

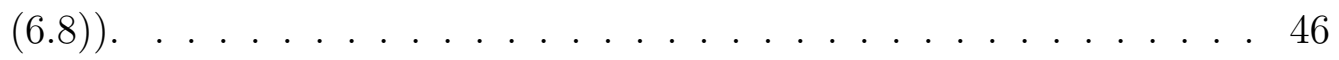


7.7 The extracted imaginary dielectric response from bulk crystalline $\mathrm{ZnO}$ as compared to the pseudo-harmonic phonon model (dashed, eq. (6.3)), the plasmon response from the modified Drude model (dots, eq. (6.6)), and the modified Drude coupled plasmon-phonon model (solid, eq. (6.8)). . . . . . . . . . . . . . . . . . 46

7.8 Extracted $\mathrm{ZnO}$ wafer refractive index compared to the pseudo-harmonic phonon model (dashed, eq. (6.3)) and the improved coupled plasmonphonon model (solid, eq. (6.8)). . . . . . . . . . . . . . . . 47

7.9 Extracted $\mathrm{ZnO}$ wafer extinction coefficient compared to the pseudoharmonic phonon model (dashed, eq. (6.3)), the modified Drude model (dots, eq. (6.6)), and the modified coupled plasmon-phonon model (solid, eq. (6.8)). . . . . . . . . . . . . . 47

7.10 Extracted $\mathrm{ZnO}$ wafer power absorption compared to the pseudoharmonic phonon model (dashed, eq. (6.3)) and the modified coupled plasmon-phonon model (solid, eq. (6.8)) . . . . . . . . . . . 48

7.11 Real (blue) and imaginary (red) components of the native conductive response as compared to the real component (solid) of the modified Drude model from eq. (6.14), and the imaginary component (dashed). 48

7.12 Experimentally measured time domain response. . . . . . . . . . . 51

7.13 Experimentally measured frequency domain response. . . . . . . . . . 51

7.14 Comparison of results extracted using the effective medium model for three different plate separations. . . . . . . . . . . . . . . 52

7.15 The extracted real dielectric response from $\mathrm{ZnO}$ nanowires as compared to the pseudo-harmonic phonon model (dashed, eq. (6.3)), and the modified Drude coupled plasmon-phonon model (solid, eq. (6.8)). 54 
7.16 The extracted imaginary dielectric response from $\mathrm{ZnO}$ nanowires as compared to the pseudo-harmonic phonon model (dashed, eq. (6.3)), the plasmon response from the modified Drude model (dots, eq. (6.6)), and the modified Drude coupled plasmon-phonon model (solid, eq.

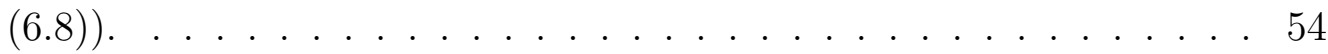

7.17 Further extraction to yield the pure $\mathrm{ZnO}$ nanowire response from within the height of the $1 \mu \mathrm{m}$ tall $\mathrm{ZnO} /$ air composite layer. . . . . . 55

7.18 The extracted extinction coefficient from pure $\mathrm{ZnO}$ nanowires as compared to the pseudo-harmonic phonon model (dashed, eq. (6.3)), the modified Drude model (dots, eq. (6.6)), and the modified coupled plasmon-phonon model (solid, eq. (6.8)) . . . . . . . . . . . . . 55

7.19 The extracted power absorption from pure $\mathrm{ZnO}$ nanowires as compared to the pseudo-harmonic phonon model (dashed, eq. (6.3)), the modified Drude model (dots, eq. (6.6)), and the modified coupled plasmon-phonon model (solid, eq. (6.8)) . . . . . . . . . . . 56

7.20 Real (blue) and imaginary (red) components of the native conductive response as compared to the real component (solid) of the modified Drude model from eq. (6.14), and the imaginary component (dashed). 56

7.21 A comparison of bulk vs. nanowire $\mathrm{ZnO}$ electrical conductivity. . . . 58

7.22 Real component of the dielectric response based on theoretic parameter fit. . . . . . . . . . . . . . . . . . . 59

7.23 Imaginary component of the dielectric response based on theoretic parameter fit. . . . . . . . . . . . . . . . . . 59 


\section{Chapter 1}

\section{Introduction}

\subsection{Thesis Statement}

The frequency dependent dielectric properties of bulk and nanowire zinc oxide $(\mathrm{ZnO})$ as determined in the terahertz region are presented in this thesis. A method for characterizing nanostructures at terahertz by use of a parallel-plate waveguide (PPWG) and an effective medium model is presented as an alternative to previously reported techniques. Experimental results obtained through terahertz time-domain spectroscopy (THz-TDS) are compared to the well established pseudo-harmonic phonon model. A modified Drude model is presented as an improvement. The frequency dependent complex conductivity is extrapolated from measurement and compared with the modified Drude model for $\mathrm{ZnO}$ in bulk and nanowire form.

\subsection{Thesis Outline}

A brief introduction to the broad field of terahertz research and why $\mathrm{ZnO}$ is a material of interest follows in this chapter.

A description of the THz-TDS equipment and basic operation can be found in Chapter 2. This includes an overview of how a THz-TDS instrument functions as well as the setup considerations for typical free-space measurements. Chapter 3 
provides a brief review of PPWG operation and details the PPWG construction of the PPWG used in this work. An introduction to zinc oxide is given in Chapter 4 followed by a detailed description of the method used to produce the $\mathrm{ZnO}$ nanowires measured in this work.

The method used to solve for the complex refractive index from a time domain waveform is described in Chapter 5. A few equations are presented to familiarize the reader with the inverse model and a simple effective medium model. A method used to evaluate measurement uncertainty in this work is also presented. Chapter 6 introduces the coupled plasmon-phonon dielectric model that will be used to infer material parameters when fit to experimental data. A discussion of the method used to extract complex conductivity follows. Chapter 7 presents the extracted material parameters from the bulk and nanowire measurements. A summary of this work is presented in Chapter 8 followed by a discussion of future work in Chapter 9.

\subsection{Terahertz}

Until recently, the electromagnetic spectrum accessible through electronics and photonics has had a gap between microwave circuits that typically operate at tens of GHz and infrared lasers that can operate down to tens of THz. Advances in semiconductor technology have pushed the realm of electronics up to $845 \mathrm{GHz}[1]$ and beyond with the indium phosphide / indium gallium arsenide heterojunction bipolar transistor (HBT). Far infrared and free electron lasers (FIR and FEL) operating down below $1 \mathrm{THz}$ in conjunction with advanced semiconductors have narrowed what has been traditionally referred to as the $\mathrm{THz}$ gap. The range of frequencies that define the terahertz regime is generally accepted to be from 0.3 to $10 \mathrm{THz}$. Definitions vary on the frequencies bounding the upper FIR and the lower MIR, but 
it is commonly accepted that FIR extends down to $0.3 \mathrm{THz}$ and MIR spans up to $100 \mathrm{THz}$.

The development of terahertz time-domain spectroscopy in recent years has made this region more readily accessible for general research. The nonlinear optic technique employs the ultrafast gating of a photoconductive switch from a femtosecond laser to generate freely propagating subpicosecond waves. These pulses are extremely broadband typically spanning several hundred $\mathrm{GHz}$ to several $\mathrm{THz}$, and have proven extremely useful for material characterization and material identification [2] applications.

The ability to generate high power in this region remains a significant challenge. Recent advances in quantum cascade lasers (QCL) have been able to produce several hundred milliwatts of power at $1.39 \mathrm{THz}$ [1]. For comparison, the average power of a typical THz-TDS system is on the order of microwatts.

The terahertz region holds many possibilities for material sensing due to the rotational and vibrational transitions many substances undergo in this region, which produce unique $\mathrm{THz}$ absorption fingerprints. The interest in spectroscopic analysis applies to a wide variety of disciplines such as security, industrial process control, agriculture, the food industry, and medical diagnostics, to name a few. Terahertz radiation does not pose extreme health risks since it is non-ionizing, which makes it an attractive alternative to X-rays for material sensing and imaging. Although $\mathrm{THz}$ is absorbed by water it can be used to image tissues of varying water content. Most plastics, textiles and paper are nearly transparent to $\mathrm{THz}$, which allows for such security applications as identifying the chemical composition as well as the shapes of concealed objects. The ability to perform non-contact measurements of submicron precision is already in practice with such applications as material degradation 
evaluation and precision dispense of layers of paint on aircraft [3].

According to Peter Siegel of the Jet Propulsion Laboratory since approximately one-half of the total luminosity and $98 \%$ of the photons emitted since the Big Bang fall into the submillimeter and far-infrared, continuous wave (CW) terahertz technology has long interested astronomers. Of interest are targets such as singly ionized nitrogen, which has a spectral line at $1461 \mathrm{GHz}$ and is only detectable by $\mathrm{CW}$

terahertz astronomy [1]. When the Cosmic Background Explorer investigated the infrared and microwave remnants of the big bang, it detected singly ionized nitrogen with crude, angular resolution. Heterodyne receivers may allow scientists to tease kinematic information from their studies of the skies to determine how stars and clusters affect the ionized interstellar medium.

\section{$1.4 \mathrm{ZnO}$}

Pulsed terahertz radiation has been demonstrated from a photoconductive switch fabricated on high-resistivity single-crystal $\mathrm{ZnO}$ [4]. $\mathrm{ZnO}$ is a promising material for terahertz optics since it has a wide band gap, high mobility and resistivity, and it is transparent over a broad frequency range. The experimental results indicated that $\mathrm{ZnO}$ exhibits a high electric field breakdown, which promises $\mathrm{ZnO}$ to have high power terahertz applications. For this reason it is essential to explore the detailed optical and dielectric properties of $\mathrm{ZnO}$ in the broad terahertz region.

Nanomaterials have been investigated for use with dye-sensitized solar cells (DSSCs) due to their high surface area as a means to transport electrons from the photo-excited dye molecules to the conducting substrate. Recent studies have suggested that dense arrays of $\mathrm{ZnO}$ nanowires could improve electron transport by 
providing direct conduction pathways to the substrate while maintaining a high surface area [5]. The conductivity of nanostructured material differs greatly from that of the bulk crystalline form, which makes measurements performed on the as-grown structures of interest highly desirable. With typical carrier scattering rates of $10^{12}$ to $10^{14} \mathrm{~s}^{-1}$ overlapping the terahertz region, THz-TDS is a desirable non-contact technique to measure conductivity. 


\section{Chapter 2}

\section{Terahertz Time-Domain Spectroscopy}

\subsection{THz-TDS System}

The terahertz system used for the THz-TDS experiments reported in this work generates and detects $\mathrm{THz}$ based on the gated photoconductive switch technique [2]. A pumped mode-locked Ti:Sapphire laser generates a pulse less than $100 \mathrm{fs}$ in duration. The beam is split before reaching the photoconductive emitter and detector antennas, with a translation stage included in the detector path introducing a time delay.

The emitter consists of a DC biased photoconductive antenna on a semiconductor substrate with high breakdown voltage, high carrier mobility, and a short carrier lifetime. The laser pulse illuminates the substrate and generates enough free carriers for the switch to close allowing for the static DC bias to accelerate the free carriers while charge density rapidly declines from carrier trapping in defect sites. The current spike that arises is the source of the subpicosecond pulses of electromagnetic radiation.

The mechanism for detection is almost the reverse of the emitter. The detector is not biased, which allows the received terahertz field to induce a current in the gap when the laser pulse excites the photocarriers. The received field amplitude is 
proportional to the induced photocurrent, and the shape is mapped out by varying the time delay between the emitter and detector pulses while measuring the current.

The system used for this work has a useful signal bandwidth from 0.2 to $3 \mathrm{THz}$ with a peak signal to noise ratio greater than $70 \mathrm{~dB}$. The $\mathrm{THz}$ field is focused down to a spot size of $3.5 \mathrm{~mm}$ with a pair of 3-inch focal lenses attached to the emitter and detector. An acrylic chamber was constructed to enclose the emitter, detector, and specimen, and purged with dry air in order to eliminate the effects of water absorption on the measurements.

The process by which material parameters are extracted in a typical THz-TDS experiment requires an analysis method for $\mathrm{THz}$ wave propagation. Typically the transmitted time-domain waveform is measured with and without a sample present and then a Fourier deconvolution is performed to extract the material response, which is then compared to a model of the frequency response of the system in order to solve for the unknown(s). Thus the method by which material parameters are extracted is to solve an inverse problem. 


\section{Chapter 3}

\section{Parallel-Plate Waveguides}

\subsection{PPWG Application}

Previous $\mathrm{THz}$ measurements of nanomaterial required greater electromagnetic field interactions than were possible on an as-grown samples. With the largest nanostructure dimension typically two orders of magnitude smaller than the shortest wavelengths excited, the phase and amplitude changes induced are less than the inherent drift and jitter in the measurement instrumentation. In an effort to overcome this

problem differential time-domain spectroscopy (DTDS) [6] and double modulation techniques [7] were developed. The previously reported methods still required either repeated growth cycles on an optically transparent substrate or scraping the nanomaterial into a silicon containment cell. Both of these methods have the potential to alter and damage the structures intended for measurement, therefore it would be preferable to perform experiments on in situ nanostructures.

A waveguide allows the electromagnetic field to laterally traverse along as long of a path of the sample as needed in order to induce a detectable phase shift. The waveguide provides the additional desirable function of containing the field along the path of propagation inside the guide. By focusing the field onto the entrance of the waveguide it is possible to concentrate a larger field density that can interact 
with the material than is possible with free space propagation. Figure 3.3 on page 14 shows a schematic cross-section of PPWG as part of TDS measurement setup.

The tradeoff involved in constricting the plates closer together is the difficulty in coupling the field into the PPWG. Additionally, the problem with spacing the plates too far apart is that multimodal propagation will be allowed. The phenomenon of multimode propagation occurs when multiple solutions to the electromagnetic boundary conditions in the PPWG exist. The reason this presents a problem is that higher mode propagation does not exhibit a linear phase relationship with frequency. The frequencies propagating at higher modes will have slower propagation velocities than those in lower modes, which will result in multiple arrival times for the same spectral content.

The terahertz emitter generates a linearly polarized electromagnetic wave. In free space operation the orientation of the field is often ignored since the transmitter and receiver are placed with the same orientation, which allows for maximum reception of the linearly polarized transverse electromagnetic (TEM) wave. No signal will be detected if the receiver is oriented at $90^{\circ}$ with respect to the transmitter. When transmitting the terahertz pulse through a PPWG the orientation of the linear polarization with respect to the plates will result in either transverse magnetic (TM) or transverse electric (TE) mode propagation inside the waveguide.

\subsubsection{Transverse Magnetic Mode}

When the polarization of the incident field is oriented perpendicular to the surface of the plates the electromagnetic boundary conditions are satisfied down to DC, hence the plates act like a standard transmission line with no low-frequency cutoff. A diagram of the E-field orientation with respect to the plates appears in Figure 3.3 
on page 14. The lowest $\mathrm{TM}_{0}$ mode is the same as TEM and is the required mode of operation for the material parameter extraction method used in this work. This requirement is due to the non-dispersive propagation supported by the $\mathrm{TM}_{0}$ mode. To satisfy the electromagnetic boundary conditions in the $\mathrm{TM}_{n}$ mode the incident electric field will be of the form:

$$
E_{y}=\left(-j \beta / k_{c}\right) A_{n} \cos (n \pi y / d) e^{-j \beta z}
$$

where the cutoff wavenumber $k_{c}$, and the propagation constant $\beta$ are:

$$
\begin{gathered}
k_{c}=\frac{n \pi}{d}, \quad n=0,1,2,3 \ldots \\
\beta=\sqrt{k^{2}-k_{c}^{2}}
\end{gathered}
$$

and the cutoff frequencies to each mode is related to the plate separation $d$ by:

$$
f_{c}=\frac{n}{2 d \sqrt{\mu \varepsilon}}
$$

The relationship between propagation constant and cutoff frequency results in dispersion for all modes higher than $n=0$. Without precise knowledge of how much frequency content is contained in each mode a model of the system cannot be made. Therefore, we restrict our investigation to frequencies in the dominant $\mathrm{TM}_{0}$ mode in this work. In another work [8] a method was proposed to determine the contributions from each mode in an overmoded PPWG, which may enable the dominant mode restriction to be relaxed in order to achieve maximum SNR through improved input coupling.

Metal shim washers were used as plate separators, which restricted $d$ to discrete 
values at roughly $100 \mu \mathrm{m}, 200 \mu \mathrm{m}, 300 \mu \mathrm{m}$, and above. The cutoff frequencies for the $\mathrm{TM}_{1}$ mode using those plate separations are $1.5 \mathrm{THz}, 750 \mathrm{GHz}$, and $500 \mathrm{GHz}$ respectively. Given that TDS system bandwidth extends to between $1.6 \mathrm{THz}$ and 2 THz the thinnest of the $100 \mu \mathrm{m}$ shims were used in the experimental measurements.

\subsubsection{Construction}

The top and bottom plates of the parallel-plate waveguide (PPWG) were machined out of $4^{\prime \prime} \times 2^{\prime \prime} \times 1^{\prime \prime}$ aluminum blocks with a $6^{\circ}$ taper milled out of each end leaving a $2^{\prime \prime}$ square planar surface in the middle of each block. The $6^{\circ}$ taper at the input and output of the PPWG was included courtesy of Alex Higgins to improve free space coupling [9]. Two inset positioning pins were placed in diagonal corners of the bottom block to be used as guideposts. In addition, screw threads were tapped in the opposite diagonal corners, which were used to secure the top block to the bottom.

The crossed guideposts and screws were used to prevent mismatches in alignment of the top and bottom blocks. Screw threads were tapped in the top and side of the blocks in order to secure the entire PPWG to the measurement platforms. This fixed the bottom plate of the waveguide so that only the top plate was exchanged between measurements, which helped minimize positioning errors between sample and reference measurements. The central $2^{\prime \prime} \times 2^{\prime \prime}$ planar area was reserved for thin film and nanowire deposition. The machined aluminum blocks were then electroplated with gold, which had the added benefit of reducing conductive attenuation losses. 


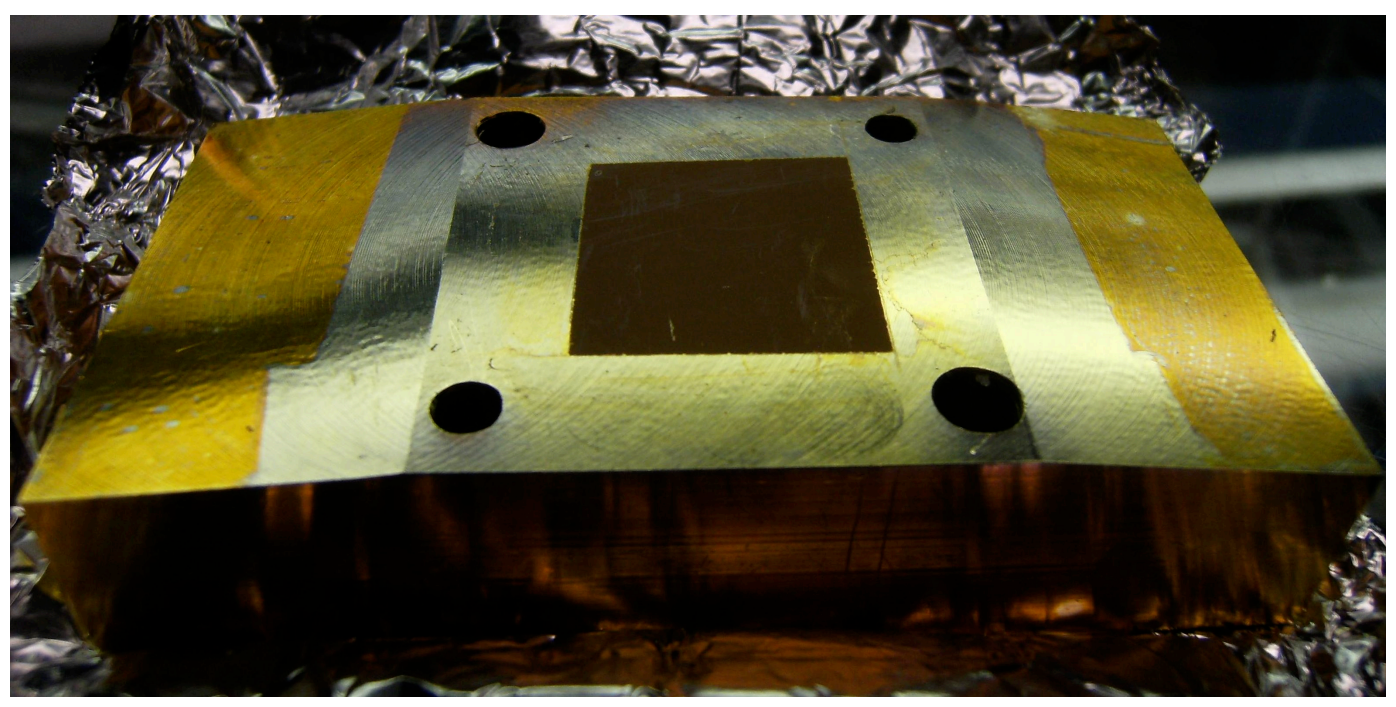

Fig. 3.1: ZnO nanowire deposition on gold plated PPWG.

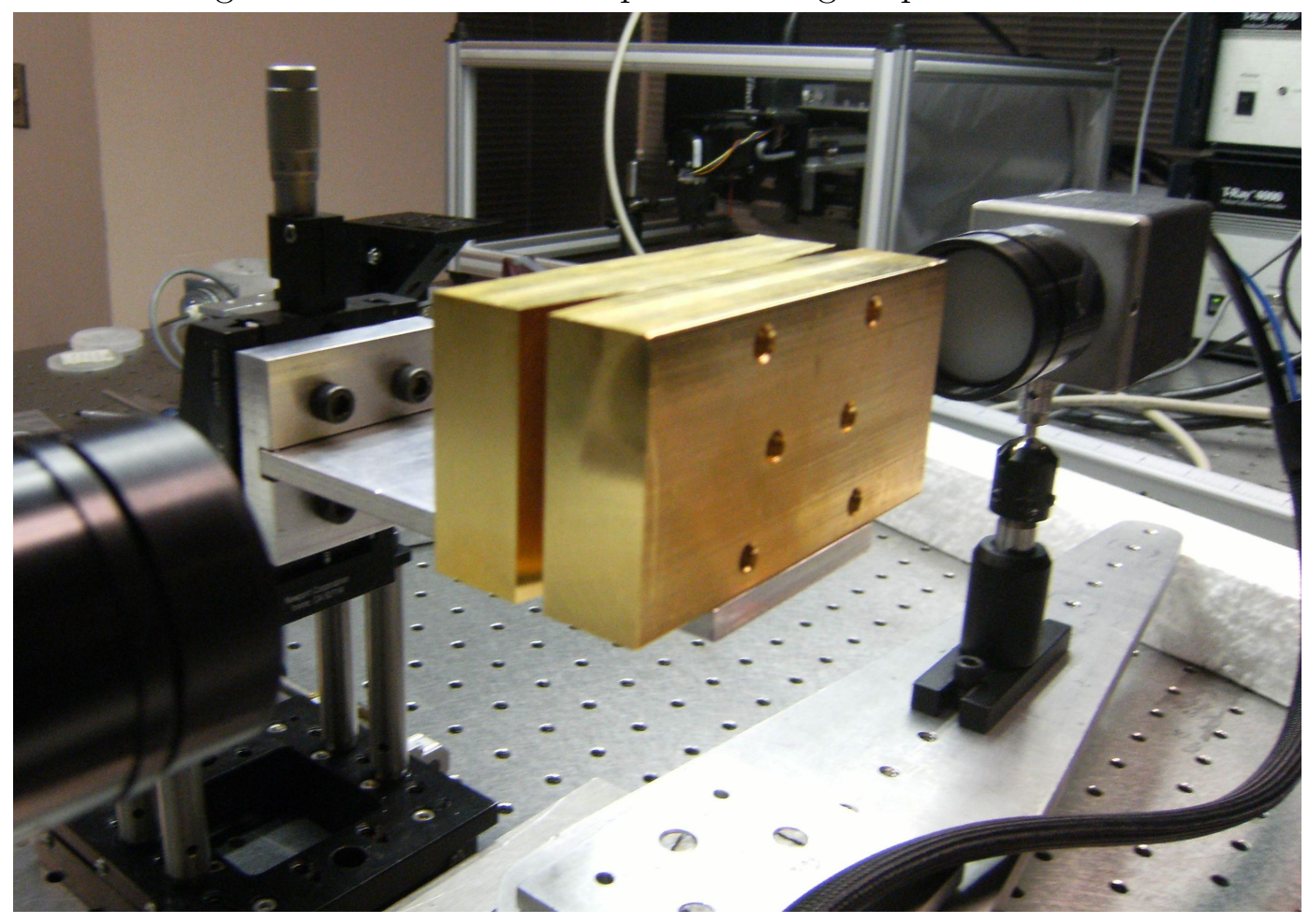

Fig. 3.2: Gold plated PPWG in experimental setup. 


\subsubsection{Experimental Setup}

When conducting free-space transmission measurements through a waveguide special care must be taken in order to avoid misalignment from the central axis. Failure to do so can result in frequency dependent amplitude and phase differences that will corrupt the extracted solution for the dielectric response.

Misalignment was avoided in the experimental setup with the use of a rotational arm to mount the receiver on. A top-down diagram of the setup appears in Figure 3.3 on page 14 and a photo taken from the side can be seen in Figure 3.2 on page 12. Once visual alignment was achieved with straight edges and levels the receiver arm was moved side to side at equal off-axis angles. Small differences in path length are readily apparent as can be seen in Figure 3.4 on page 14 and fine adjustments can then be made to line up the receiver along the central axis. 


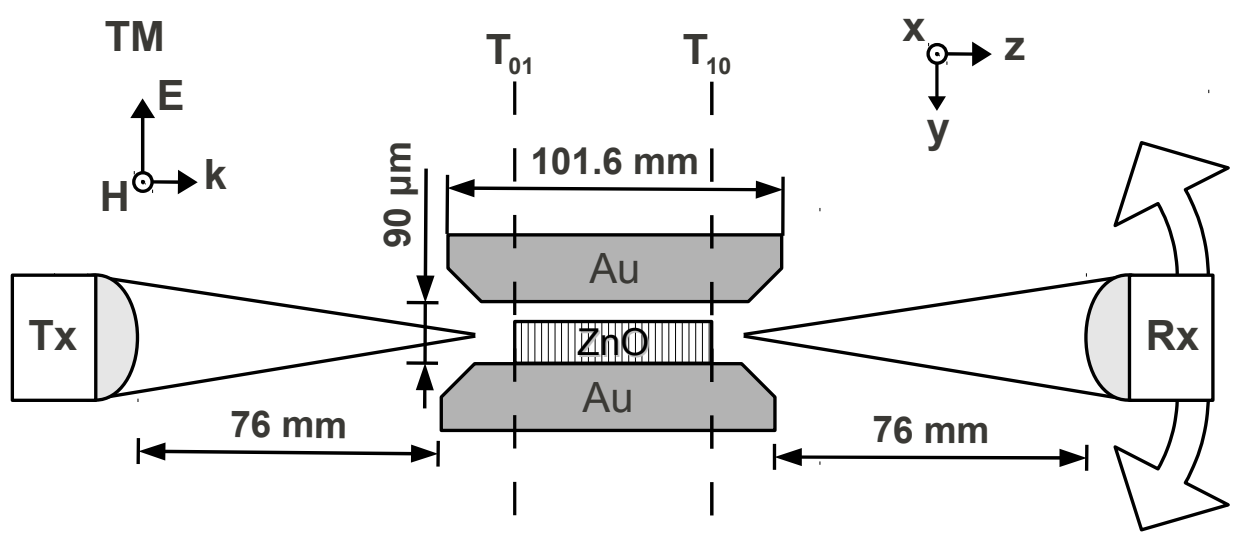

Fig. 3.3: Diagram of the PPWG experimental setup.

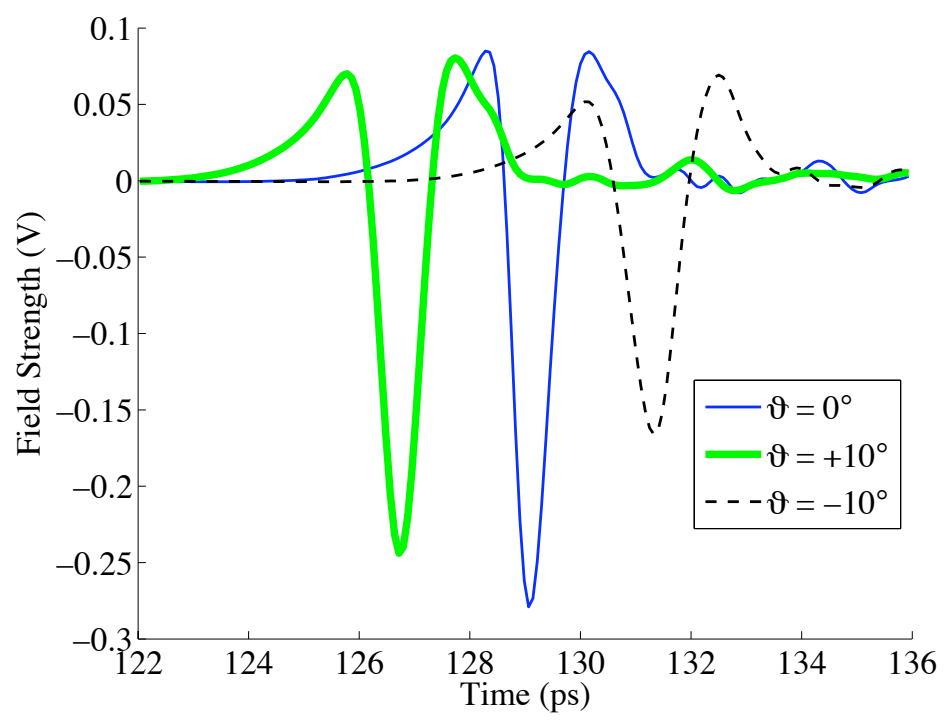

Fig. 3.4: Using the THz-TDS system to detect misalignment. 


\section{Chapter 4}

\section{Zinc Oxide}

\subsection{Properties}

Zinc oxide is a II-VI semiconductor with a direct band gap of $\sim 3.37 \mathrm{eV}$ at room temperature. ZnO most commonly crystallizes in the wurtzite structure and has a refractive index of approximately 2.8. The effective mass of the electron in $\mathrm{ZnO}$ is commonly accepted to be $0.24 m_{e}$, and it is reported to have a large free-exciton binding energy of about $60 \mathrm{meV}[10]$. This high exciton energy has laser applications since it allows excitonic emission processes to persist even above room temperature. The lack of control over its electrical conductivity has hindered its use as a semiconductor, with $\mathrm{ZnO}$ crystals tending to always be $n$-type. Obtaining reproducible low-resistivity $p$-type doping in $\mathrm{ZnO}$ has been a notoriously difficult task. However, $p$ - $n$ heterostructures can be made by depositing $n$-type $\mathrm{ZnO}$ films on other $p$-type materials [11]. An interesting attribute of $\mathrm{ZnO}$ is that with proper doping it can be made electrically conductive while maintaining transparency in the visible region, which allows for transparent electrical contacts.

ZnO exhibits large piezoelectric constants and has a sensitive surface to the presence of absorbed species, lending itself useful in a variety of sensing applications. $\mathrm{ZnO}$ has strong non-linear resistive and optical properties. ZnO crystals and thin 
films exhibit second- and third-order non-linear optical behavior, a necessary characteristic for photoconductive substrates used in THz emission and detection [10]. The exceptionally high radiation hardness it possesses makes it an attractive material for space applications [11]. Interestingly, it has recently been reported [12] that $\mathrm{ZnO}$ exhibits birefringence at $\mathrm{THz}$ along the $<10 \overline{1} 0>$ orientation.

The bulk material measurements presented in this work were obtained from an undoped $\mathrm{ZnO}$ single crystal wafer with $<0001>$ orientation. It should be noted that birefringence does not occur when propagating $\mathrm{THz}$ normal to this orientation. The $10 \times 10 \mathrm{~mm}$ wafer was reported to be $0.5 \mathrm{~mm}$ thick and had both sides polished resulting in a surface roughness of $\leq 10 \AA$. A hydrothermal growth method was used, which yields a wafer of high resistivity and excellent crystalline quality. Resistivity, carrier mobility and carrier concentrations obtained from a 2 inch hydrothermally

grown $\mathrm{ZnO}$ wafer [13] were reported to be $380 \Omega \cdot \mathrm{cm} \pm 15 \%, 200 \mathrm{~cm}^{2}(V \cdot s)^{-1} \pm 10 \%$, and $8 \times 10^{13} \mathrm{~cm}^{-3} \pm 20 \%$, respectively.

\subsection{Nanowire Growth}

High quality, vertically aligned $\mathrm{ZnO}$ nanowires had been successfully grown by Athavan Nadaraja using the chemical bath technique on ITO, FTO/glass, and silicon wafer substrates at the time this work was proposed [14]. However, for measurements to take place in a parallel-plate waveguide $\mathrm{ZnO}$ nanowires needed to be grown on a metal substrate. Initially, attempts were made to produce nanowires directly onto the aluminum blocks being used to construct the waveguide.

An image obtained from a Zeiss sigma scanning electron microscope (SEM) that appears in Figure 4.1 on page 18 revealed the morphology to primarily consist of a dissolved non-crystalline thin film interspersed with micron wide $\mathrm{ZnO}$ crystal rods. 
An attempt to anodize a small aluminum block resulted in crystalline structures over the areas that anodized after chemical deposition, which indicated the aluminum was reacting strongly with the oxygen in the chemical bath and needed to be suppressed. The results of this attempt appear in Figure 4.2 on page 18. Experimenting with a $\mathrm{ZnO}$ thin layer deposited on aluminum to act as a buffer and seed layer produced mixed results. As can be seen in Figures 4.3 and 4.4 on page 19 nanowire growth was achieved but a significant portion was deformed due to aluminum contamination.

A successful growth of $\mathrm{ZnO}$ nanowires on gold sputtered silicon was carried out courtesy of Athavan Nadaraja in a low temperature aqueous bath using a solution of zinc nitrate hexahydrate and hexamethylenetramine [15]. The gold sputtered wafer was coated with a planar ZnO thin layer by spray pyrolysis prior to growth [16]. The spray pyrolysis solution contained $0.05 \mathrm{M}$ of zinc acetate in ethanol with a small amount of acetic acid to lower the $\mathrm{pH}$. Repeating this process on an aluminum waveguide plate electroplated with gold was once again successful, the results of which can be seen in an SEM image that appears in Figure 4.5 on page 20. A macro image of the same deposition appears on page 12 in Figure 3.1.

In order to estimate nanowire height a sample was grown on gold sputter coated silicon and broken in half. Although slightly difficult to tell from the SEM image in Figure 4.7 on page 21 a survey of multiple areas revealed the average nanowire height to be about $1 \mu \mathrm{m}$. 


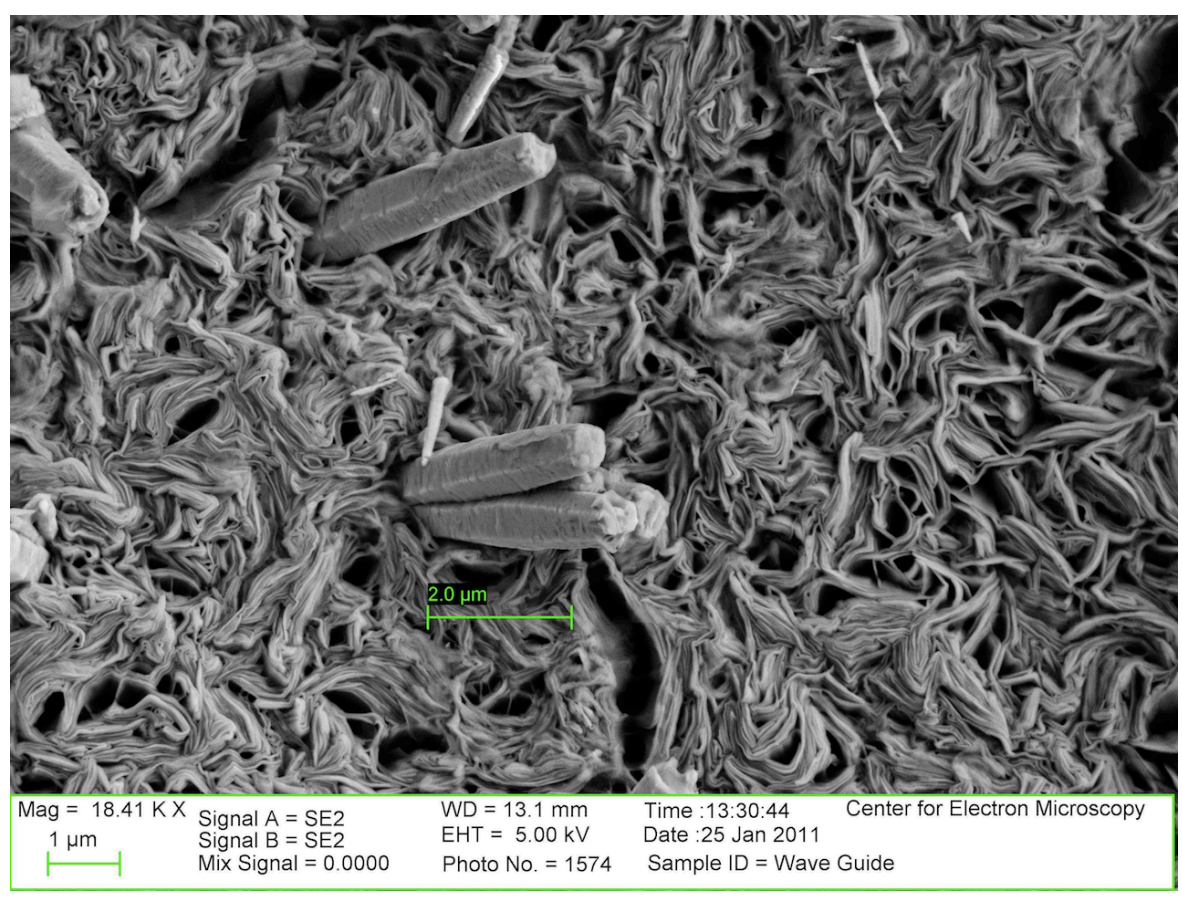

Fig. 4.1: SEM image of the first attempt at $\mathrm{ZnO}$ nanowire growth on aluminum.

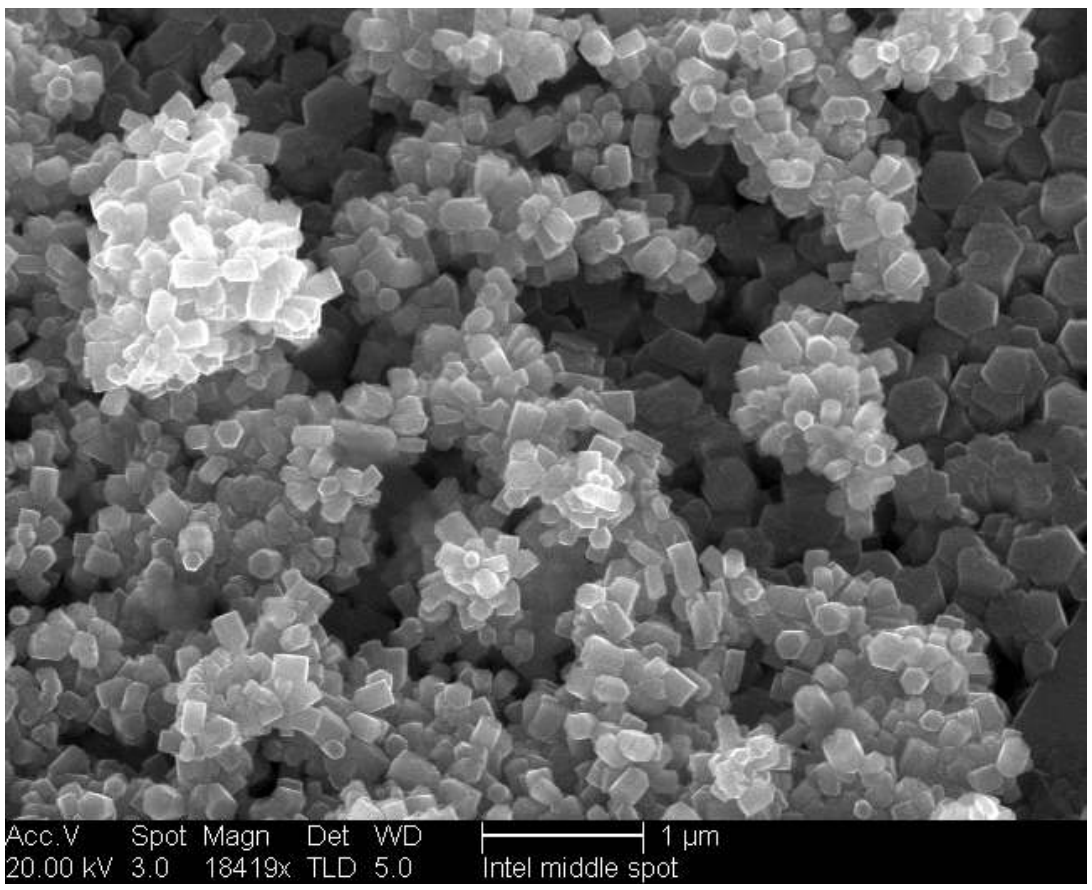

Fig. 4.2: SEM image of the second attempt at $\mathrm{ZnO}$ nanowire growth on anodized aluminum. 


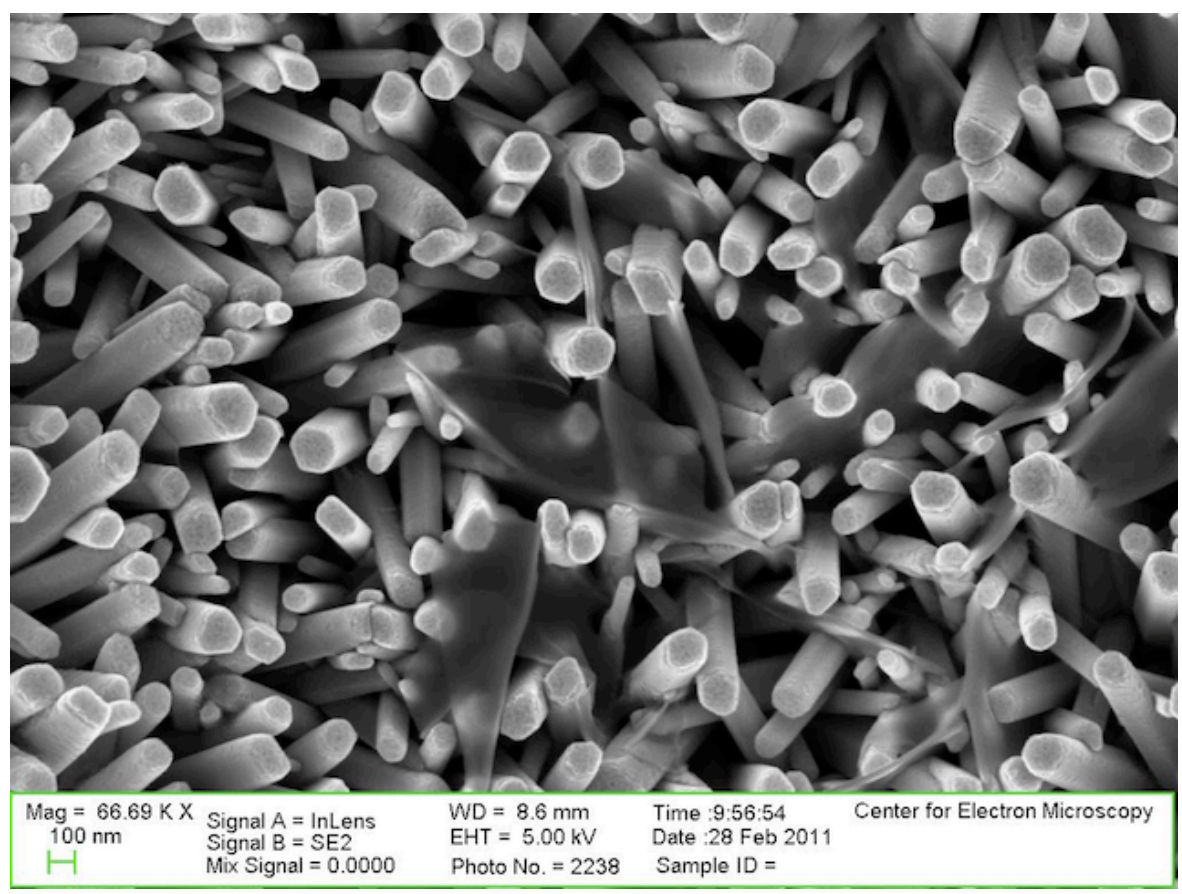

Fig. 4.3: SEM image of the third attempt at $\mathrm{ZnO}$ nanowire growth on top of a thin film layer on aluminum.

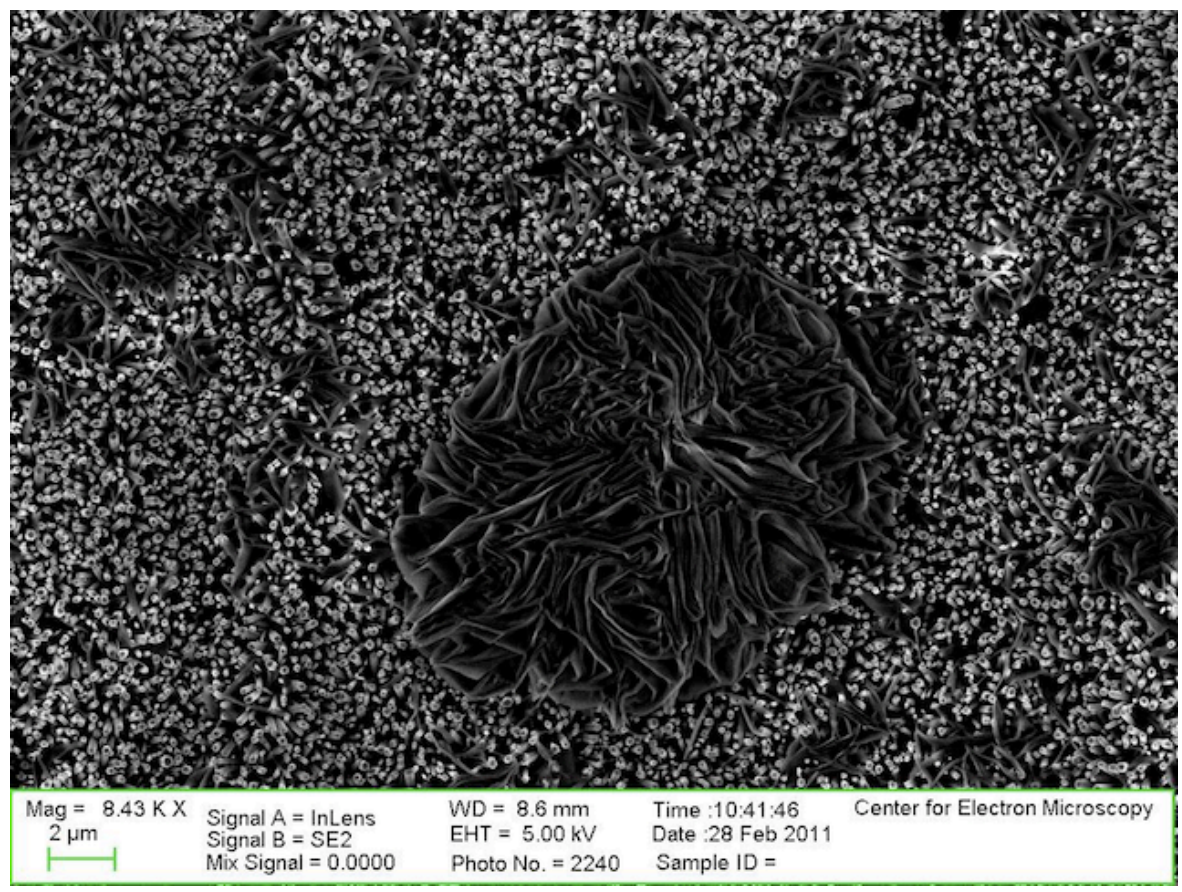

Fig. 4.4: Aluminum deformations interspersed in $\mathrm{ZnO}$ nanowires. 


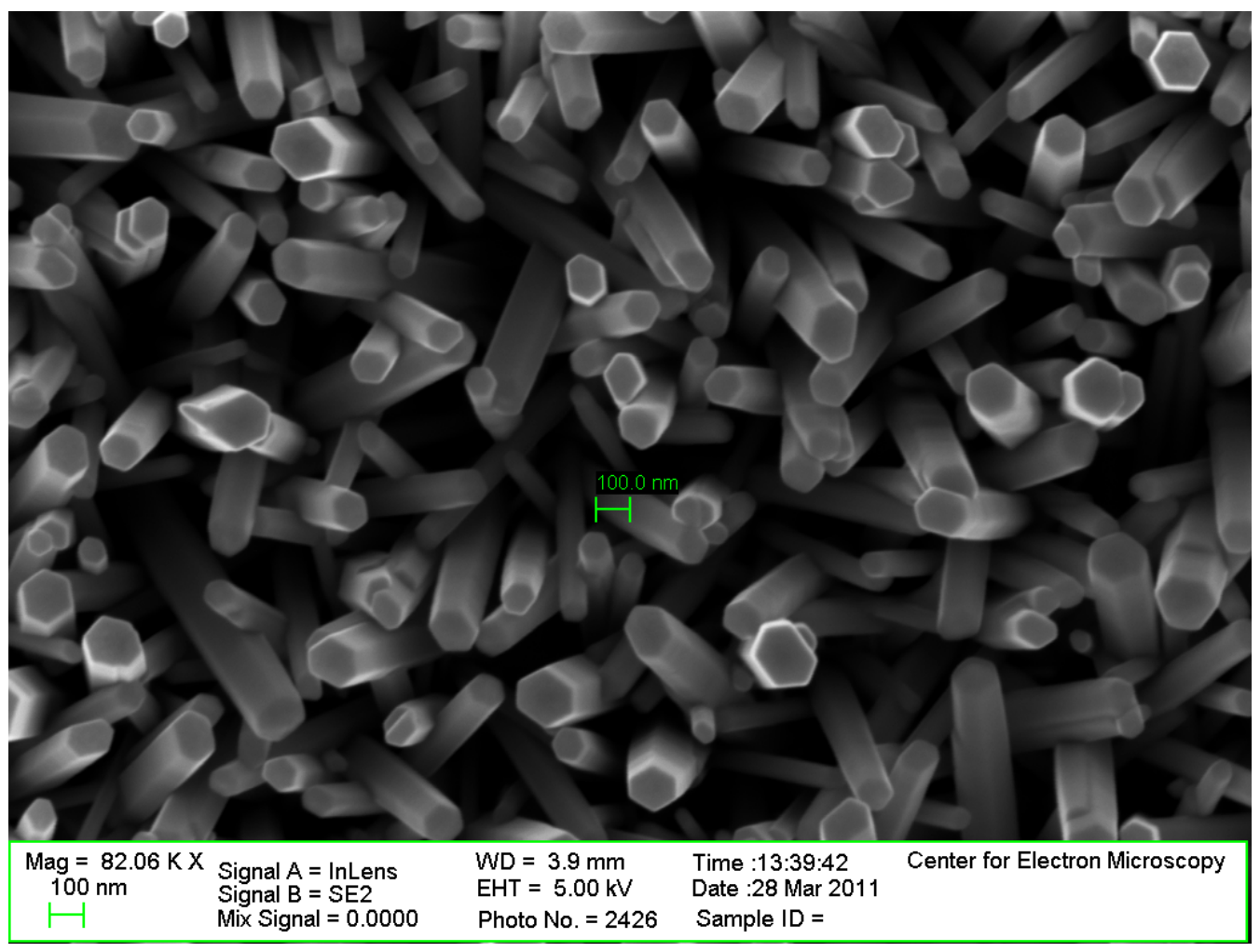

Fig. 4.5: SEM image of the successful $\mathrm{ZnO}$ nanowire growth on gold plated aluminum.

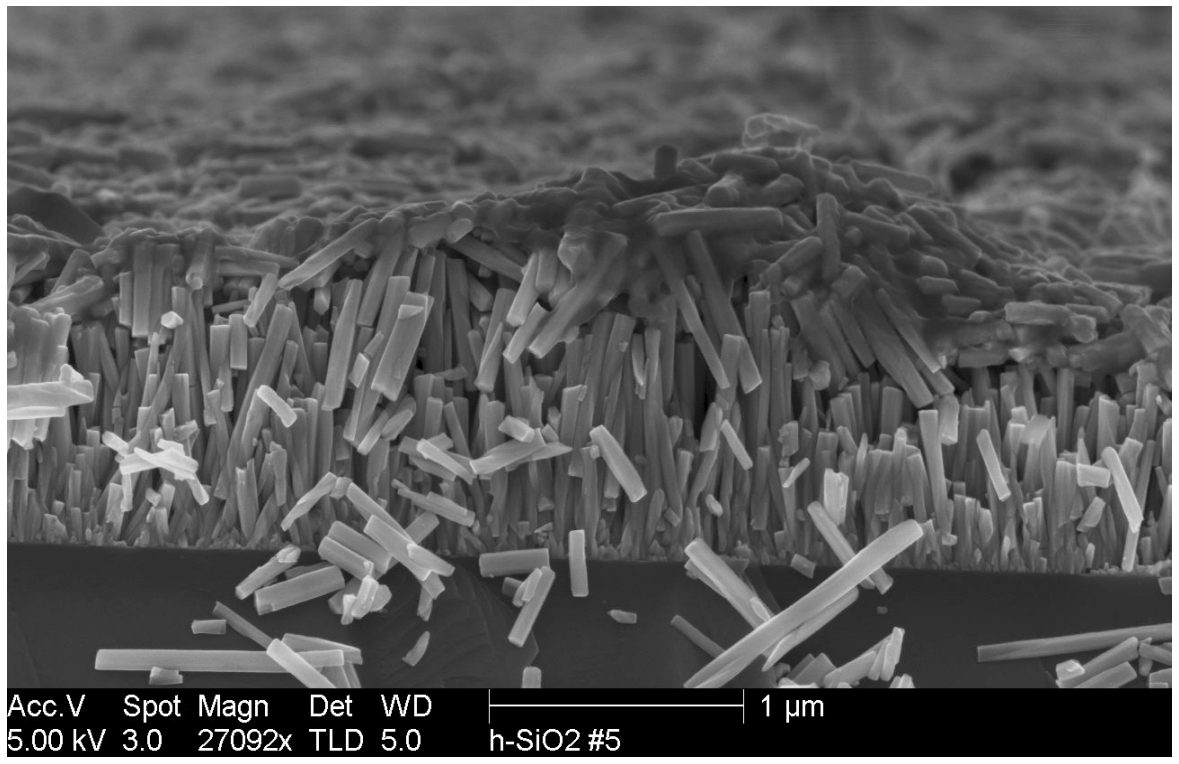

Fig. 4.6: SEM image of $\mathrm{ZnO}$ nanowires grown on gold sputtered silicon. 


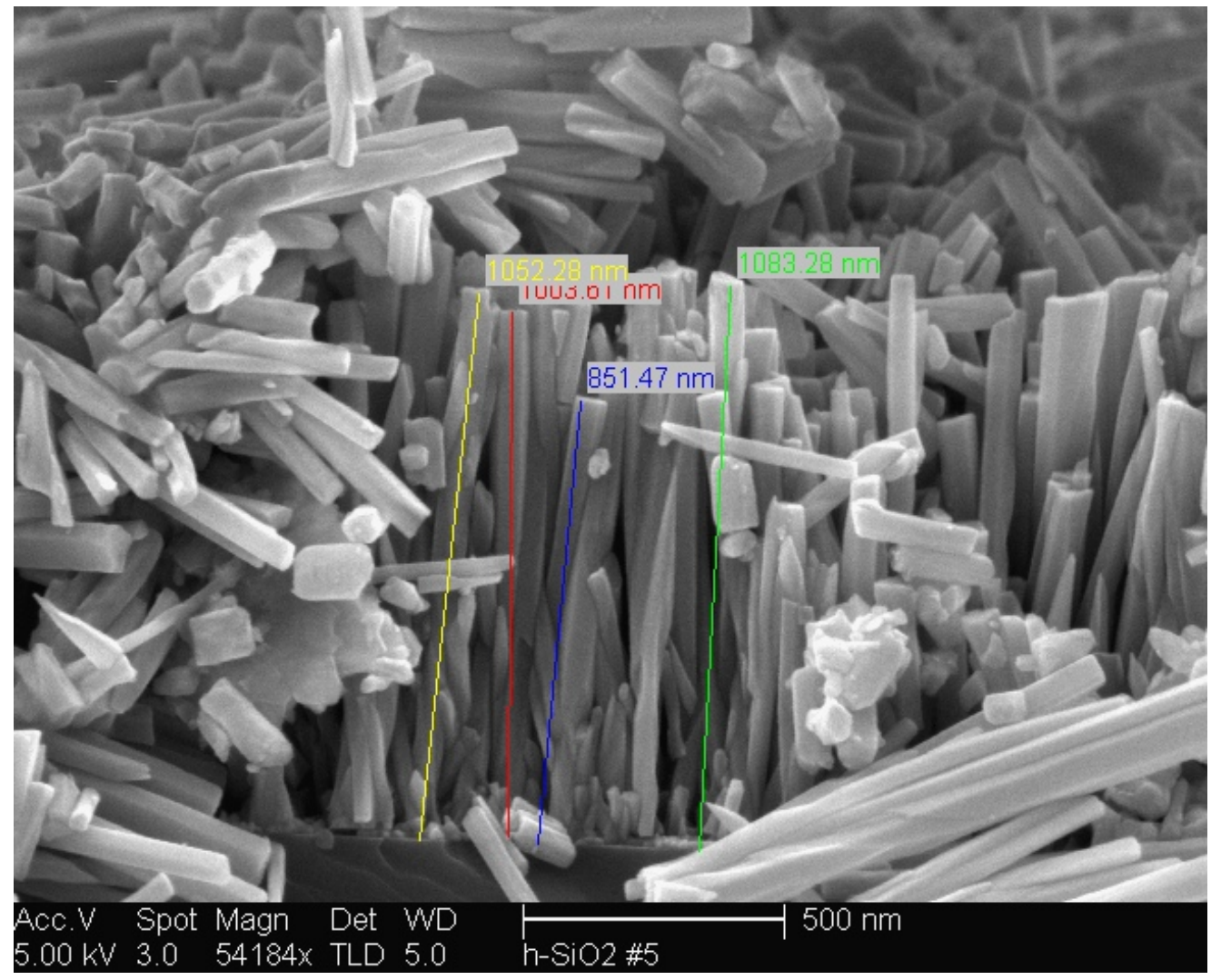

Fig. 4.7: Height measurement from an SEM image of $\mathrm{ZnO}$ nanowires grown on gold sputtered silicon. 


\section{Chapter 5}

\section{Material Parameter Extraction}

\subsection{Inverse Model}

\subsubsection{Free space}

Inverse model extraction methods require a reference waveform. In the case of bulk materials the reference is propagated through free space. Thin films may be characterized in a method similar to the free space technique by using a bare substrate of the same thickness as that supporting the thin film as the reference [6]. Whatever the case may be, the method of extracting the complex refractive index requires sample and reference measurements be deconvolved by dividing the discrete Fourier Transform of the sample response from the reference waveform.

$$
H_{\text {experiment }}(\omega)=\frac{\overline{E_{\text {samp }}^{e x}}(\omega)}{\overline{E_{\text {ref }}^{e x}}(\omega)}
$$

Next, a transfer function of the deconvolved system must be derived. As long as the phase of the measured and modeled deconvolutions extrapolate to zero at zero frequency, a numerical solver can be applied to find a unique solution.

Measuring the free space propagation of a $\mathrm{THz}$ pulse through a wafer can be complicated by the presence of internal reflections that will produce ripple in the 


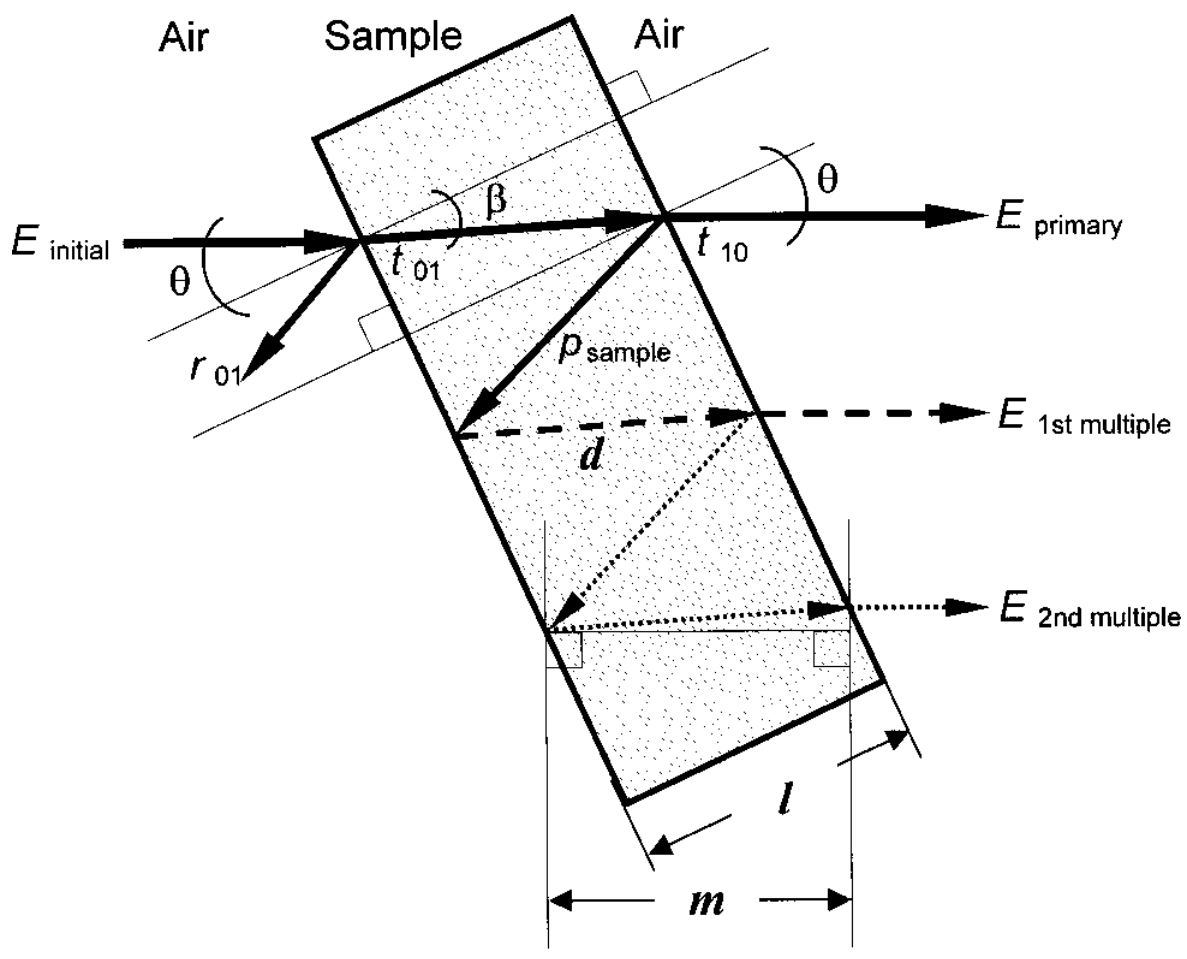

Fig. 5.1: Inverse model diagram [17].

frequency response, the results of which appear in Figures 7.1 and 7.2 on page 42 . A diagram of wave propagation through a sample that includes multiple reflections appears above in Figure 5.1. Most commonly a simplified model that assumes normal incidence is presented in the literature, but a slight angle off the normal accounted for in the solution [17] can be included in order to minimize ripple in the extracted material parameters as much as possible. The deconvolved transfer function that includes a primary transmission and 2 subsequent multiples through the sample will be

$$
H(\omega)=T_{01} T_{10} \times\left(\exp \left\{\frac{-j d \omega\left[\tilde{n}(\omega)-\tilde{n}_{a i r}\right]}{c}\right\}\right) \times F P(\omega)
$$




$$
F P(\omega)=\left\{1+\sum_{k=1}^{2}\left[R_{10}^{2} \exp \left(\frac{-j d \omega \tilde{n}(\omega)}{c}\right)^{2}\right]^{k}\right\}
$$

where $F P(\omega)$ represents multiples due to internal reflection, a process also known as the Fabry-Perot effect. $T_{01}, T_{10}$, and $R_{10}$ represent the frequency dependent Fresnel transmission and reflection coefficients at the material to air interface as defined in eqs. (5.4) and (5.5), $c$ is the speed of light and $\tilde{n}_{\text {air }}$ is given to be $1.00027+i 0$ for dry air.

$$
\begin{aligned}
T_{01}(\omega) & =\frac{2 \tilde{n}_{0}(\omega) \cos \theta}{\tilde{n}_{0}(\omega) \cos \beta+\tilde{n}_{1}(\omega) \cos \theta} \\
R_{10}(\omega) & =\frac{\tilde{n}_{0}(\omega) \cos \theta-\tilde{n}_{1}(\omega) \cos \beta}{\tilde{n}_{1}(\omega) \cos \beta+\tilde{n}_{0}(\omega) \cos \theta}
\end{aligned}
$$

The solution set of the refractive index is dependent on the sample thickness $l$ and incident angle $\theta$ provided. The two parameters define the path length through the sample through the relation $d=l / \cos \beta$.

Solutions obtained from deconvolved systems that include multiple reflections are highly sensitive to thickness error. This sensitivity can be used as a tool to estimate the actual thickness of the sample that can then be used to obtain the most accurate solution. The best estimate for the thickness is found in the solution set with the least deviation across the windowed frequency range of data for which confident results have been obtained. This metric as presented by Dorney et al. $[17]$ is defined as the minimum total variation $(T V)$ from the sum of differences $(D)$ between subsequent frequency points in the solution.

$$
\begin{gathered}
D[m]=|n[m-1]-n[m]|+|\kappa[m-1]-\kappa[m]| \\
T V=\sum D[m]
\end{gathered}
$$


It should be noted that this method relies on the assumption that the complex refractive index does not vary much from one frequency sample to the next. This is because the sampled frequency step size is relatively small as compared to the spectral features from the majority of solid materials. In this work the temporal window used for the bulk $\mathrm{ZnO}$ measurement was approximately 100 ps long with a sample rate of $74 \mathrm{fs}$, which resulted in a sampled frequency step size of $\Delta f \cong 10$ $\mathrm{GHz}$.

\subsubsection{Waveguide}

The PPWG extraction method requires an empty reference waveguide with the same plate separation, position, and alignment as that of the waveguide containing the sample. Restricting wave propagation to the lowest order $\mathrm{TM}_{0}$ mode simplifies the wave propagation model inside the PPWG to a TEM wave. Any coupling and conductive attenuation losses are assumed to be the same as that in the reference, and therefore those terms cancel in the measured and modeled deconvolution. The path length through the PPWG is long enough that internal reflections do not produce temporally adjacent multiples, which reduces the model to a single pass through an effective dielectric medium with the following transfer function:

$$
H(\omega)=T_{01} T_{10} \times\left(\exp \left\{\frac{-j l \omega\left[\tilde{n}_{e f f}(\omega)-\tilde{n}_{a i r}\right]}{c}\right\}\right)
$$

where $l$ is the length of the $\mathrm{ZnO}$ nanowire patch traversed and $\tilde{n}_{e f f}$ is the effective complex refractive index that results from the simultaneous propagation through the $\mathrm{ZnO}$ nanowire/air composite layer and remaining air that spans the height of the layer to the other waveguide plate. As illustrated in Figure 3.3 on page 14, $T_{01}$ and $T_{10}$ represent the transmission coefficients into and out of the effective medium 
that occurs inside the PPWG.

\subsection{Effective Medium Model}

In this investigation we treat the composite of $\mathrm{ZnO}$ and air within the height of the nanowire layer as the pure medium of interest and the air filled space between the top of the nanowires and the upper waveguide plate as the host medium. A simple mixing model can then be used to describe the distribution of material inside the waveguide,

$$
\varepsilon_{e f f}(\omega)=f \varepsilon_{\mathrm{m}}(\omega)+(1-f) \varepsilon_{\mathrm{h}}
$$

where $\epsilon_{e f f}, \epsilon_{h}$, and $\epsilon_{m}$ are respectively the dielectric constants of the effective medium, the host medium, and the pure medium of interest. The filling factor is defined as the ratio of pure to host medium. From this simple effective medium model the material parameters of interest can be isolated from the remaining empty waveguide by defining the fill factor as the ratio of pure medium height to waveguide plate separation. Note that the simple effective medium model is the best choice when one medium is considerably smaller than the other. Given the relation $\epsilon=\epsilon_{r}+i \epsilon_{i}=\left(n_{r}+i n_{i}\right)^{2}=\tilde{n}^{2}$ the complex refractive index of the pure medium can be found after substitution into eq. (5.9) to be:

$$
\tilde{n}_{\mathrm{m}}(\omega)=\sqrt{\frac{\tilde{n}_{e f f}^{2}(\omega)-(1-t / d) \cdot \tilde{n}_{\mathrm{h}}^{2}(\omega)}{t / d}} .
$$

The nanowire height is $t$, and $d$ is the separation distance between waveguide plates. The frequency dependent power absorption is related to the imaginary term in the complex refractive index by

$$
\alpha(\omega)=\tilde{n}_{\mathrm{i}}(\omega) 2 \omega / c .
$$


It should be noted that negative imaginary term solutions to (5.10) are discarded since positive $\Im\left\{\tilde{n}_{\mathrm{m}}\right\}$ corresponds to positive absorption.

\subsection{Measurement Uncertainty}

Uncertainty in THz-TDS experiments should always be taken into account since a typical measurement is the result of thousands of averages, which are taken over a period of time in which some unknown amount of drift and amplitude variation occurred in the measurement instrumentation. This means that as the signal-tonoise ratio (SNR) falls into single digits there can be significant variation in the solution to the inverse problem. Previous investigations into the limits of time averaging on the THz-TDS system revealed no significant improvement from oneminute to ten-minute averages, and the difference between sequential ten-minute averages always revealed the presence of drift. For this work a single minute-long average over 6000 measurements taken every 10 ms was considered sufficient.

Measurements the $\mathrm{THz}$ system are primarily affected by thermal noise in the detector, phase noise in the optical time delay stage, and amplitude variations due to fluctuations in laser light intensity. A measurement taken with no signal present revealed the detector noise to be frequency independent. This noise was classified as additive white noise. Variations from measurement to measurement not correlated with white noise are assumed to be independent from the additive white noise and are classified as frequency dependent statistical error. The method of calculating the impact of white noise and frequency dependent statistical error was presented in part by Ioachim Pupeza et al. [18] and appears in the appendix. The result, as applied to the 6000 individual measurements used in this work, is a measure of variation in the deconvolved transfer function. 
The general rule of thumb for determining the acceptable bandwidth over which an average of measurements can yield a meaningful result when fed to a numerical solver is the range in which the unwrapped phase linearly extrapolates to zero at DC. However, this method is subject to the best judgement of the user since one may choose to present data extracted from measurements whose linearly unwrapped phase trends to zero at DC but contains a number of outliers. For this reason an effort was made in this work to address measurement uncertainty.

The manner in which measurement confidence was evaluated in this work was to define the upper frequency limit as the point at which $|\Delta H(\omega)| \geq|H(\omega)|$, the results of which appear in Figures 5.2 and 5.3 on page 29. For PPWG measurements there is an additional uncertainty if one extends the frequency range past the $\mathrm{TM}_{1}$ cutoff, as determined by eq. (3.4) on page 10. If the phase of the deconvolved measurement has little deviation past the cutoff frequency this method will actually serve to slightly extend the upper bound of the frequency range. As can be seen in Figure 5.3 this method extended the upper bound of the useful frequency range slightly past the cutoff frequency of $1.6 \mathrm{THz}$ an extra $66 \mathrm{GHz}$. The maximum deviation over 6000 averages for the magnitude and phase of the bulk and nanowire measurements appear in Figures 5.4 - 5.7 on pages 30 and 31.

Unfortunately, there is no statistical method to determine the lower bound of the frequency range if the limiting factor is low frequency rejection in the experimental setup. The lower bound for the freespace and PPWG measurements were determined to be the frequency below which the extracted results appeared to significantly deviate from the normal response. 


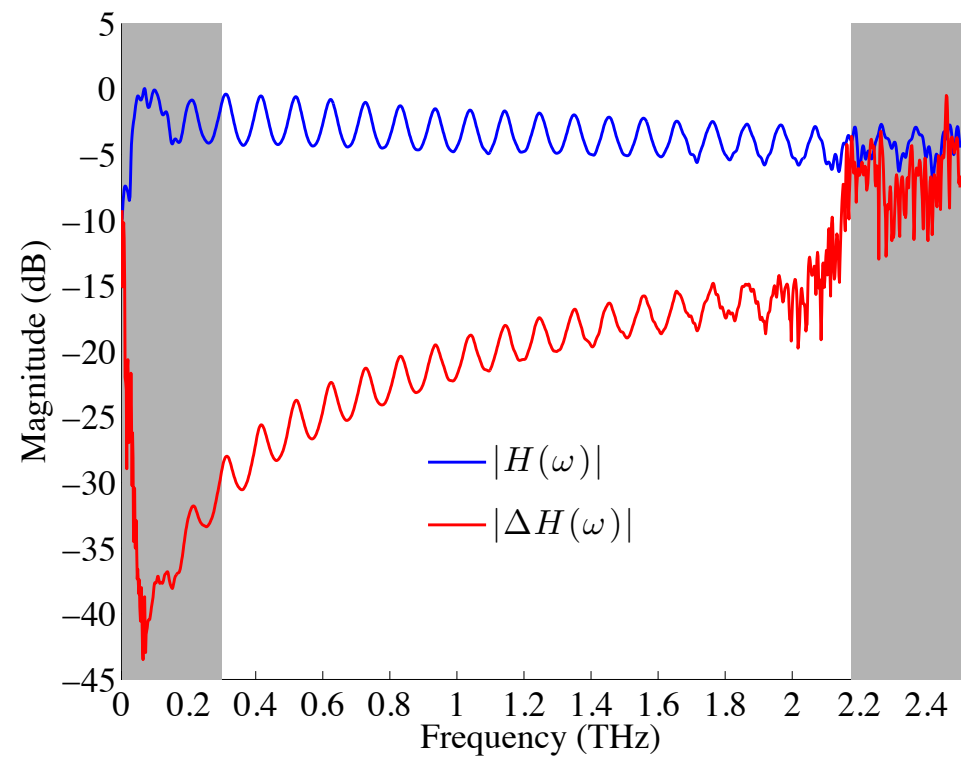

Fig. 5.2: Frequency span over which reliable results were obtained for bulk $\mathrm{ZnO}$. Interference from the fixture used to hold the bulk $\mathrm{ZnO}$ wafer may be a contributing factor at the lower bound, as can be seen in Figures 7.6 and 7.7.

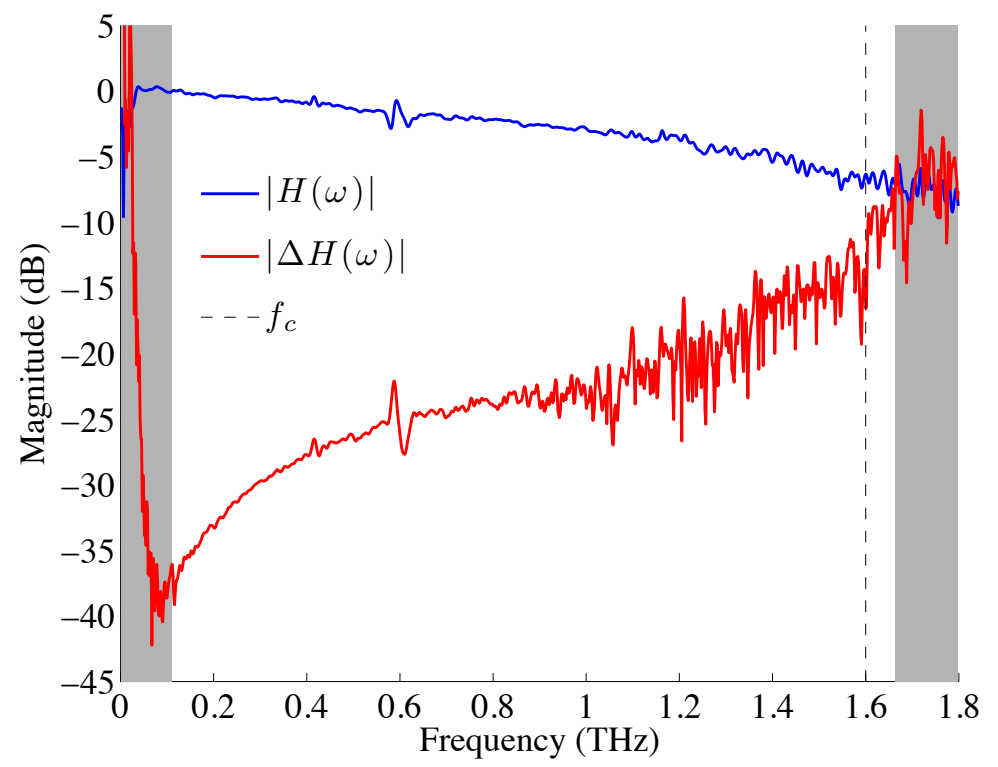

Fig. 5.3: Frequency span over which reliable results can be obtained for $\mathrm{ZnO}$ nanowires in a PPWG. 


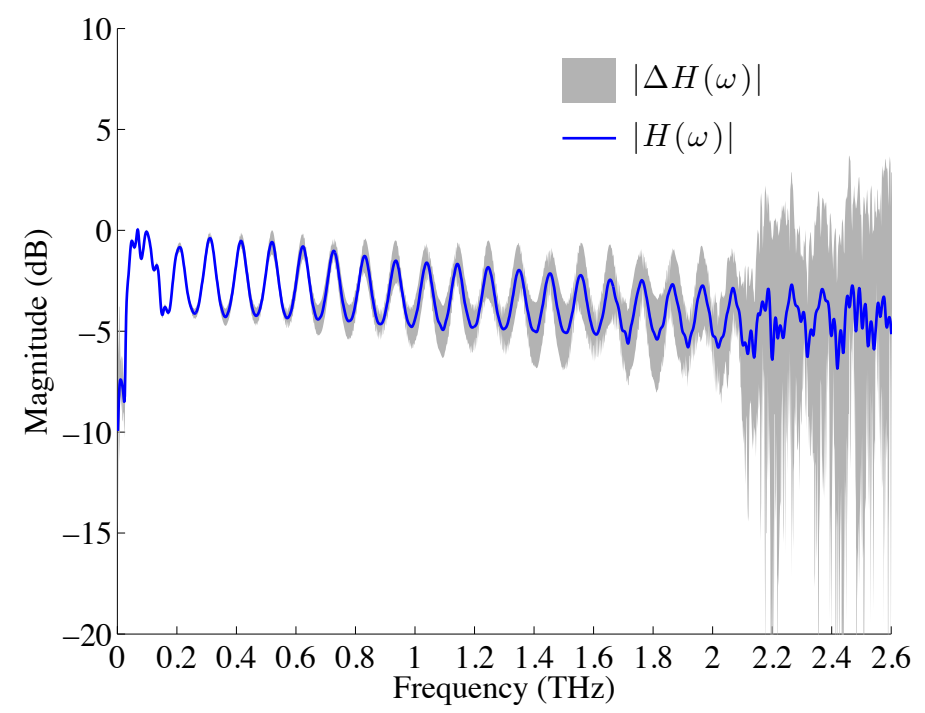

Fig. 5.4: Maximum deviation in the magnitude response of Bulk $\mathrm{ZnO}$.

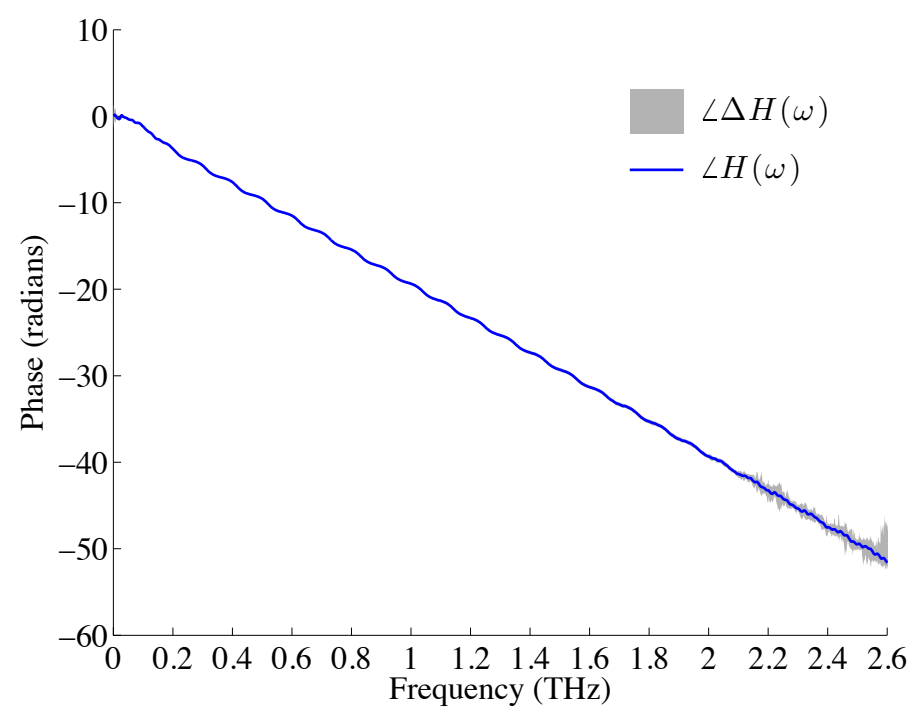

Fig. 5.5: Maximum deviation in the phase response of Bulk $\mathrm{ZnO}$. 


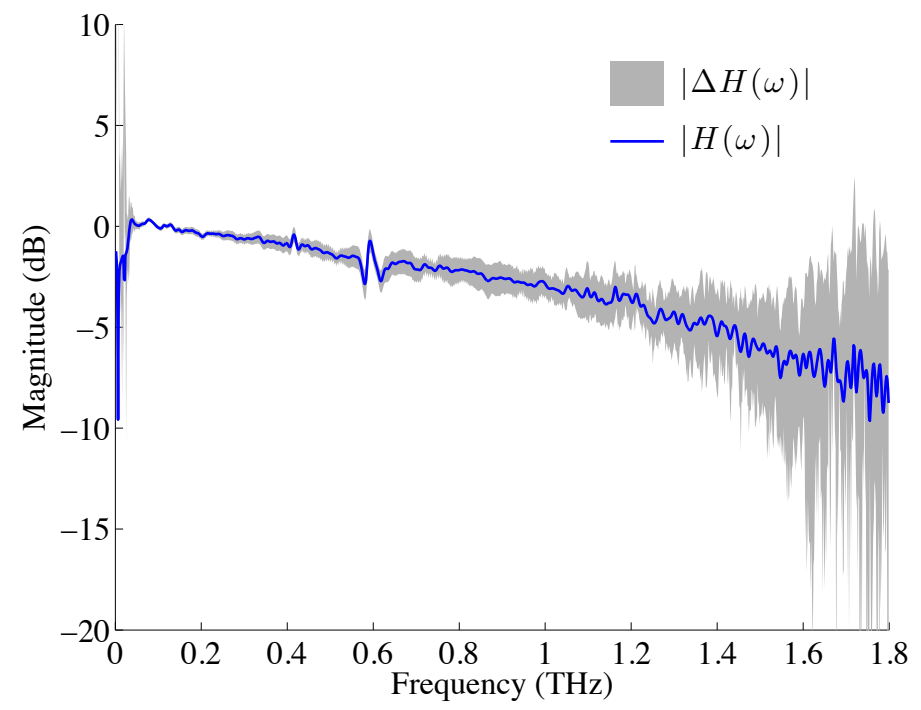

Fig. 5.6: Maximum deviation in the magnitude response of $\mathrm{ZnO}$ nanowires.

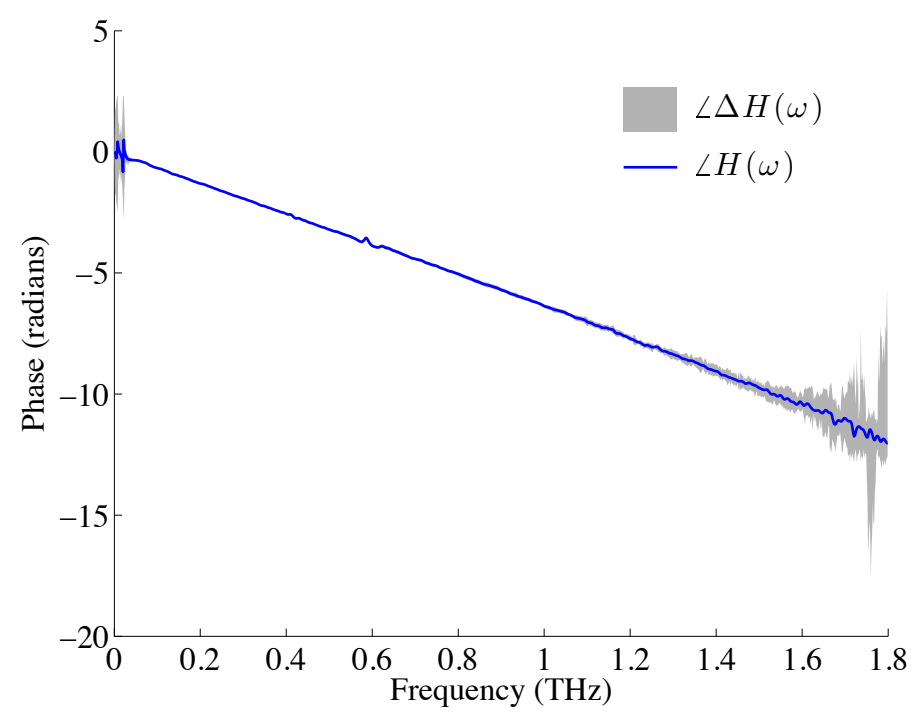

Fig. 5.7: Maximum deviation in the phase response of $\mathrm{ZnO}$ nanowires. 


\section{Chapter 6}

\section{Dielectric Theory}

\subsection{Dielectric Model}

The optical response of a material can be completely described by its dielectric function, $\varepsilon(\omega)$. The significant contributors that determine the optical behavior of an intrinsic semiconductor are the lattice response, the free electron response, and any interband transitions between energy states available to the electrons. As long as these processes do not strongly interact with each other the dielectric function $\varepsilon(\omega)$ may be divided into independent parts that describe the various physical mechanisms [19] as shown:

$$
\varepsilon(\omega)=\varepsilon_{\text {plasmon }}(\omega)+\varepsilon_{\text {phonon }}(\omega)+\varepsilon_{\text {debye }}(\omega)+\varepsilon_{\text {interband }}(\omega)
$$

Since wavelengths in the terahertz regime are far below the fundamental absorption edge of most semiconductors the inclusion of interband transitions can be neglected. For $\mathrm{ZnO}$ this absorption edge occurs near ultra-violet light. Other factors that may influence the dielectric response are the presence of impurities and defects, as well as thermal effects.

The dielectric response of a material in the terahertz regime consists primarily 
of contributions from bound electrons, plasmons, and optical phonons. These factors contribute to the coupled plasmon-phonon model (CPP), which is sometimes referred to as the total dielectric function [20].

$$
\varepsilon_{\mathrm{CPP}}(\omega)=\varepsilon_{\infty}-\frac{\omega_{\mathrm{p}}^{2}}{\omega^{2}+i \gamma \omega}+\sum_{j} \frac{\varepsilon_{\mathrm{st}_{\mathrm{j}}} \omega_{\mathrm{TO}_{\mathrm{j}}}^{2}}{\omega_{\mathrm{TO}_{\mathrm{j}}}^{2}-\omega^{2}-i \Gamma_{j} \omega}
$$

The high frequency dielectric $\epsilon_{\infty}$ describes the contribution due to bound electrons, the second term describes the contribution from conduction free electrons or plasmons, and the last term represents the contribution from lattice vibrations or optical phonons. Individual parameters are discussed below.

As is generally the case for ionic crystals, optical absorption in the far-infrared terahertz region can be attributed to lattice vibrations. The interaction of the radiated field with the fundamental lattice vibration then plays a dominant role resulting in the absorption of electromagnetic waves due to the creation or annihilation of lattice vibration. Such a process is described by the pseudo-harmonic approximation of the dielectric function [21],

$$
\varepsilon_{\text {phonon }}(\omega)=\varepsilon_{\infty}+\frac{\varepsilon_{\mathrm{st}} \omega_{\mathrm{TO}}^{2}}{\omega_{\mathrm{TO}}^{2}-\omega^{2}-i \Gamma \omega}
$$

where $\omega_{T O}$ is the transverse optical phonon frequency, $\Gamma$ is the phonon damping constant, and $\epsilon_{s t}$ is the oscillator strength connected to the high-frequency dielectric constant $\varepsilon_{\infty}$, and the low frequency dielectric constant $\epsilon(0)$ through the relation $\epsilon_{s t}=\epsilon(0)-\epsilon_{\infty}$. The phonon damping constant $\Gamma$ is a measure of the $\omega_{T O}$ resonant peak linewidth.

The square of the refractive index measured at lower frequencies will approach the static dielectric constant. The high-frequency dielectric constant is not directly 
observable through $\mathrm{THz}$ measurements and it is related to the transverse and longitudinal optical phonon frequencies and the static dielectric constant through the Lyddane-Sachs-Teller relation [19] as follows:

$$
\frac{\varepsilon(0)}{\varepsilon_{\infty}}=\left(\frac{\omega_{\mathrm{LO}}}{\omega_{\mathrm{TO}}}\right)^{2}
$$

The physical significance of $\omega_{\mathrm{LO}}$ and $\omega_{\mathrm{TO}}$ is that these are the longitudinal and transverse optical phonon frequencies with zero wave number supported by the crystal lattice. These optical phonon frequencies can be measured with Raman spectroscopy or Infrared spectroscopic ellipsometry and are well reported for bulk crystalline ZnO [22]. Generally, the static dielectric constant is obtained from infrared spectroscopic ellipsometry measurements [11] and the high-frequency dielectric constant is calculated from $\omega_{\mathrm{TO}}$ and $\omega_{\mathrm{LO}}$ mode frequencies.

Random thermal variations can delay the response of the dielectric medium to applied fields by slowing down the reorientations of the dipole moments in the material. This response is known as Debye relaxation and its process can be modeled as

$$
\varepsilon_{\text {Debye }}(\omega)=\varepsilon_{\infty}+\frac{\varepsilon_{\text {st }}}{1-i \omega \tau_{D}}
$$

where the Debye relaxation time $\tau_{D}$ has typical time scales of microseconds to nanoseconds at room temperature [23]. Since the inclusion of this effect only dominates the dielectric response at very low frequencies it is often neglected when modeling $\mathrm{THz}$ measurements. In this work the inclusion of the Debye relaxation into the dielectric function was explored but it was ultimately dropped since it proved ineffective at modeling any of the experimental measurements. 
Previous THz-TDS characterizations of $\mathrm{ZnO}$ in bulk crystalline and nanostructured forms [24] indicate there may still exist a greater experimentally measured absorption and dielectric response below $1 \mathrm{THz}$ than the pseudo-harmonic phonon model predicts. Though the free carrier contribution may be small, it may be significant enough to influence the dielectric response. In this work it was found that the inclusion of the classic Drude free carrier contribution into a coupled plasmonphonon model, as indicated in eq. (6.2) failed to account for the observed discrepancies. The contribution to the dielectric function at carrier concentration levels above $10^{14} \mathrm{~cm}^{-3}$ began to deviate significantly from the experimental results.

A modified Drude model (MDM) was originally presented to describe results found from infrared reflectivity measurements of reduced rutile titanium dioxide $\left(\mathrm{TiO}_{2}\right)$ [25]. This modified Drude model was again [26] found to best predict the plasmon-phonon behavior of $\mathrm{TiO}_{2}$ nanopowder from infrared reflectivity measurements. These findings are relevant to $\mathrm{ZnO}$ since $\mathrm{TiO}_{2}$ has a similar band structure. It was proposed that electron carriers from lattice defects are responsible for a plasmon mode that couples with the longitudinal-optical (LO) phonon modes. Baumard and Gervais derived a new dielectric model from the factorized form (also known as the four parameter model) that allows the decoupling of the LO excitations into LO phonon and LO plasmon [25]. Once decoupled the Drude description of the plasmon contribution is modified to contain a damping factor at the plasma frequency $\gamma_{\mathrm{pl}}$, as well as the static carrier damping factor at zero frequency $\gamma_{0}$ :

$$
\varepsilon_{\mathrm{MDM}}(\omega)=\varepsilon_{\infty}-\frac{\omega_{\mathrm{p}}^{2}-i \omega\left(\gamma_{\mathrm{pl}}-\gamma_{0}\right)}{\omega^{2}+i \omega \gamma_{0}}
$$

This model collapses to the standard Drude model when the plasma damping factor equals the carrier damping factor. The plasma frequency, $\omega_{\mathrm{p}}$, determines which 
frequencies can freely propagate through a material. This property is a function of carrier concentration and effective electron mass and is defined as follows:

$$
\omega_{\mathrm{p}}^{2}=\frac{N_{\mathrm{e}} e^{2}}{\epsilon_{0} m^{*}}
$$

where $N_{\mathrm{e}}$ is the electron density, e is electron charge, $\epsilon_{0}$ is free space permittivity, and $m^{*}$ is the effective electron mass. Radiation will propagate through the material when $\omega \gg \omega_{\mathrm{p}}$, and will be absorbed and reflected when $\omega \ll \omega_{\mathrm{p}}$. However, this generalization is made with the assumption that the thickness of the material is much larger than the wavelength of the radiation [27]. For broadband terahertz measurements on high resistivity material this assumption is not always satisfied since the typical wafer thickness is on the same order of magnitude as the wavelength content in the transmitted pulse.

Near the plasma frequency free electrons become displaced from the ionic lattice, which cause electric fields to build up in order to restore charge neutrality. In response the electrons then overshoot and oscillate around their original positions. At material thicknesses on the order of and below the plasma wavelength the ionic lattice may no longer be able to generate large enough fields to restore charge neutrality. The electrons would then respond with a damped oscillation about their original position resulting in a relaxation in the dielectric response, which may offer an alternative explanation for the plasma carrier-damping factor. Though the physical mechanism behind the plasma damping factor $\gamma_{\mathrm{pl}}$ is a subject for future work, in this work the observed dielectric response is well described by its inclusion into the coupled plasmon-phonon model. It should be noted that the complete model derived by Baumard and Gervais included LO terms in the phonon model. Since 
optical phonon absorption only occurs in the transverse modes for THz-TDS transmission measurements the LO terms are dropped. What remains is the modified Drude coupled plasmon-phonon model (MD-CPP):

$$
\varepsilon_{\mathrm{MD}-\mathrm{CPP}}(\omega)=\varepsilon_{\infty}-\frac{\omega_{\mathrm{p}}^{2}-i \omega\left(\gamma_{\mathrm{pl}}-\gamma_{0}\right)}{\omega^{2}+i \omega \gamma_{0}}+\frac{\varepsilon_{\mathrm{st}} \omega_{\mathrm{TO}}^{2}}{\omega_{\mathrm{TO}}^{2}-\omega^{2}-i \Gamma \omega}
$$

This model will be compared with the traditionally used pseudo-harmonic phonon model in the results section in order to give the reader an idea of why the MD-CPP model was presented. It is important to note that the manner in which parameter fits were found in this work was to visually inspect the theoretic curve with the extracted complex refractive index. Although an algorithm designed to solve for the best fit parameters would be useful, as has been developed in other work on material parameter estimation [28], it was not the focus of this work.

\subsection{Complex Conductivity}

The free carrier contribution to the dielectric function describes how charges in a material respond to an externally applied field. In a material in which the free carrier contribution dominates, the dielectric function can be determined to be

$$
\varepsilon_{\mathrm{fc}}(\omega)=\varepsilon_{\infty}+\frac{i \tilde{\sigma}}{\omega \epsilon_{0}}
$$

where the complex conductivity, $\tilde{\sigma}$, is derived from the Drude model:

$$
\tilde{\sigma}(\omega)=\frac{\sigma(0)}{1-i \omega \tau}
$$


The average electron scattering time is $\tau$, and the DC electrical conductivity, $\sigma(0)$, is

$$
\sigma(0)=N_{\mathrm{e}} e^{2} \tau / m^{*} .
$$

Note that the carrier damping factor $\gamma$ used in the model for the dielectric response due to free carriers is inversely related to the average time between electron scattering events. The free carrier dielectric response is found in the first two terms of eq. (6.2) on page 33, and is obtained by substituting eq. (6.10) into eq. (6.9) and using the $\gamma=1 / \tau$ relation.

Extracting the native electrical conductivity becomes problematic when the dielectric response is dominated by optical phonon scattering. The conductive response is often extracted by comparing different states of excitation [29]; this is because the optical phonon and high frequency dielectric response will remain unchanged allowing those terms to cancel. What remains in the dielectric function is the contribution due to additional electrons in the conduction band, and eq. (6.10) can usually predict the conductive response with good results.

An extraction of the native conductive response from a single measurement may be possible once the lattice and high frequency dielectric contributions are well understood. In this work the native complex conductivity is extracted from the experimentally measured refractive index by removing the optical phonon and high frequency bound electron contributions in the following manner,

$$
\tilde{\sigma}(\omega)=-i \epsilon_{0} \omega\left(\tilde{n}_{\mathrm{m}}^{2}(\omega)-\varepsilon_{\infty}-\varepsilon_{\text {lattice }}(\omega)\right) .
$$

In the case of nanomaterials it has been suggested that the increased surface area will lead to confinement effects [30] and carrier backscatter mechanisms [5] that 
may significantly alter the carrier decay rates. Real conductivities with a non-DC maximum or negative imaginary conductivities are often observed in nanomaterials but are not reproducible in the Drude model. To account for this a modification was proposed by Smith [31]. The Drude-Smith model is given to be

$$
\tilde{\sigma}(\omega)=\frac{\epsilon_{0} \omega_{\mathrm{p}}^{2} \tau}{1-i \omega \tau}\left[1+\sum_{n=1}^{\infty} \frac{c_{n}}{(1-i \omega \tau)}\right] .
$$

As with the summation in the last term of the dielectric model in eq. (6.2), the summation in the Drude-Smith model is often simplified to the first order case, with $c_{1}$ taken to be a value between 0 and -1 representing the expectation value of the cosine of the scattering angle. It is claimed that this term describes the persistence of velocity after the first scattering event [30].

A study of $\mathrm{ZnO}$ nanowire and thin film conductivity [5] reported that negative imaginary conductivity cannot be attributed to the effective medium model since it was also observed for thin films. Similarly, in this work a negative imaginary conductivity was observed for both the nanowires and the bulk crystalline wafer. However, the Drude-Smith model was abandoned once the modified Drude model proved to also reproduce negative imaginary conductivity. The extracted conductivity from eq. (6.12) could then be modeled by including the plasma damping factor into the Drude Model. Using the relation $\tau_{\mathrm{pl}}=1 / \gamma_{\mathrm{pl}}$, the conductivity becomes

$$
\tilde{\sigma}(\omega)=\frac{\sigma(0)+i \omega \epsilon_{0}\left(1-\frac{\tau_{0}}{\tau_{\mathrm{pl}}}\right)}{1-i \omega \tau_{0}}
$$




\section{Chapter 7}

\section{Experimental Results}

\subsection{Bulk $\mathrm{ZnO}$}

Bulk $\mathrm{ZnO}$ measurements were carried out on a $1 \mathrm{~cm}$ square by $0.5 \mathrm{~mm}$ thick wafer. Focal lenses with a $5 \mathrm{~mm}$ spot size and 3 inch focal lengths were necessary in order to reduce the beam width below the cross-sectional size of the sample. The position of the tool used to hold the sample were fixed in place during the reference measurement in order to reduce diffractive effects that may differ from those caused by the sample holder in the bulk $\mathrm{ZnO}$ measurement. The entire assembly was then enclosed in a chamber and purged with dry air.

The time and frequency domain responses of the free space reference as compared to the wave that has propagated through the $\mathrm{ZnO}$ wafer appear in Figure 7.1 on page 42. As can be seen in the $\mathrm{ZnO}$ time domain response a second and third transmitted pulse can be observed that have internally reflected and traversed three and five times the width of the wafer respectively. Although additional multiples are not noticeable with respect to the scale it is presented, in Figure 7.1 the time window allows for a total of five multiple reflections.

The spectral content in the transmitted pulses in Figure 7.2 on page 42 reveals a significant etalon effect from 0.2 to $2.2 \mathrm{THz}$. The response taken through the $\mathrm{ZnO}$ 
wafer differs from the freespace response until around $2.8 \mathrm{THz}$, after which both signals become buried in the noise. The results of the investigation into measurement uncertainty appear in Figure 5.2 on page 29, which placed the upper limit of reliable results near $2.2 \mathrm{THz}$.

Material parameters derived from sample measurements that include multiple reflections were used to get a more accurate measurement of sample thickness. In order to minimize etalon artifacts in the extracted complex refractive index a computational sweep of results derived for different thicknesses and incident angles was performed and evaluated according to eq. (5.6) and eq. (5.7) on page 24. The solutions appear in Figures 7.3 and 7.4 on page 43.

Even with these highly accurate estimates of thickness and incident angle the extracted material parameters still had small residual oscillations due to alignment mismatches resulting in slight constructive and destructive phase fronts at the receiver. One can avoid these etalon artifacts in the extracted material parameter curves by simply only including the primary transmitted wave in the windowed time domain measurement. Doing so tends to limit the bandwidth of the extracted results from samples that pass closely spaced multiples. For this investigation multiple reflections are included to yield the most broadband results. However, the residual etalon oscillation artifacts in the extracted results can be smoothed out by using a spatially moving average filter [18]. The smoothing technique was not applied in this work in order to avoid any ambiguity in the presentation of experimentally measured data as compared to parameter fit theoretic models.

The experimentally measured refractive index of bulk $\mathrm{ZnO}$ was found to be approximately 2.82, which can be found in Figure 7.8 on page 47. A gradual increase in the real dielectric response beyond $1.2 \mathrm{THz}$, as indicated in Figure 7.6 on page 


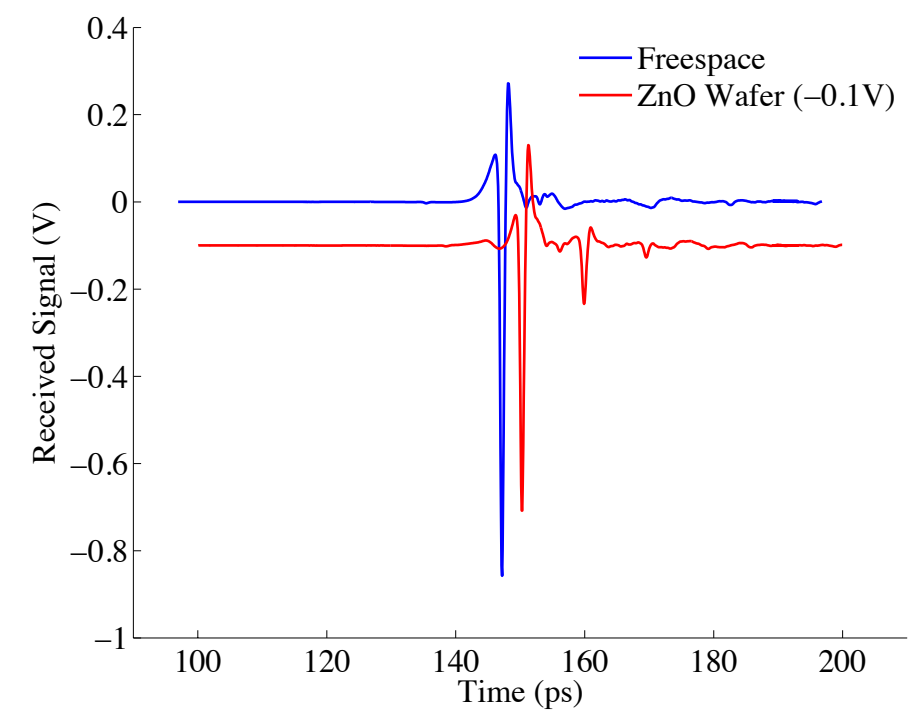

Fig. 7.1: Experimentally measured time domain response.

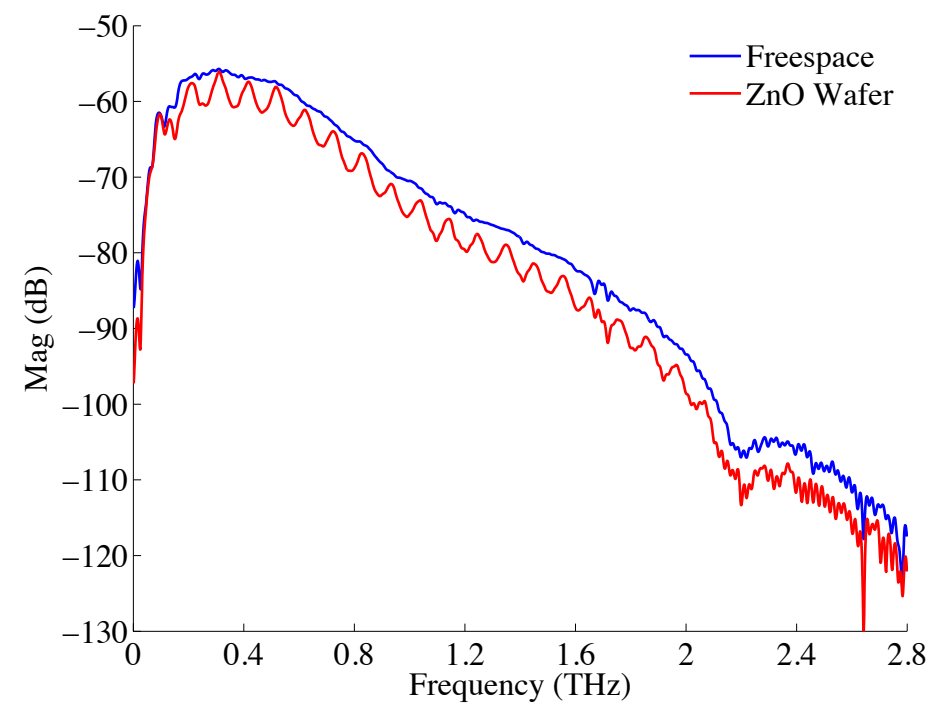

Fig. 7.2: Experimentally measured frequency domain response. 


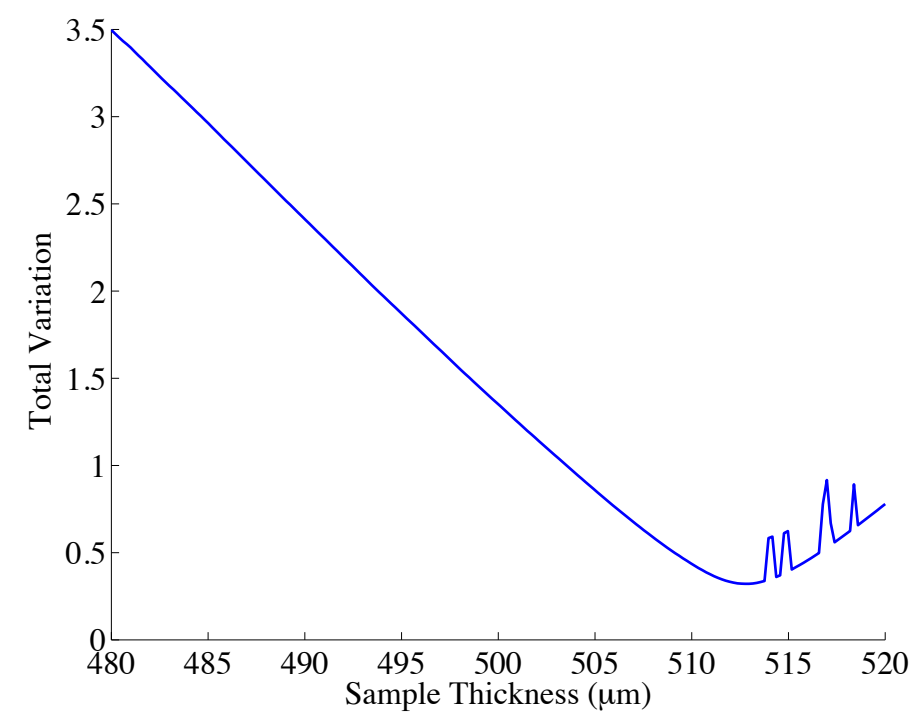

Fig. 7.3: The total variation method as applied to the $\mathrm{ZnO}$ wafer to determine actual vs. nominal thickness.

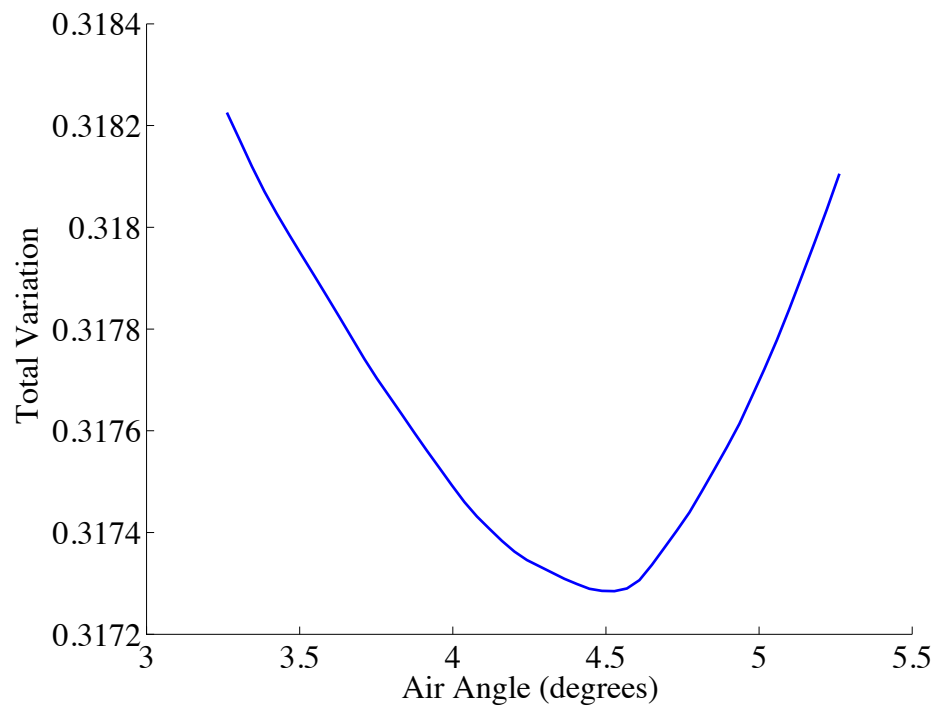

Fig. 7.4: Experimentally determined angle of incidence 


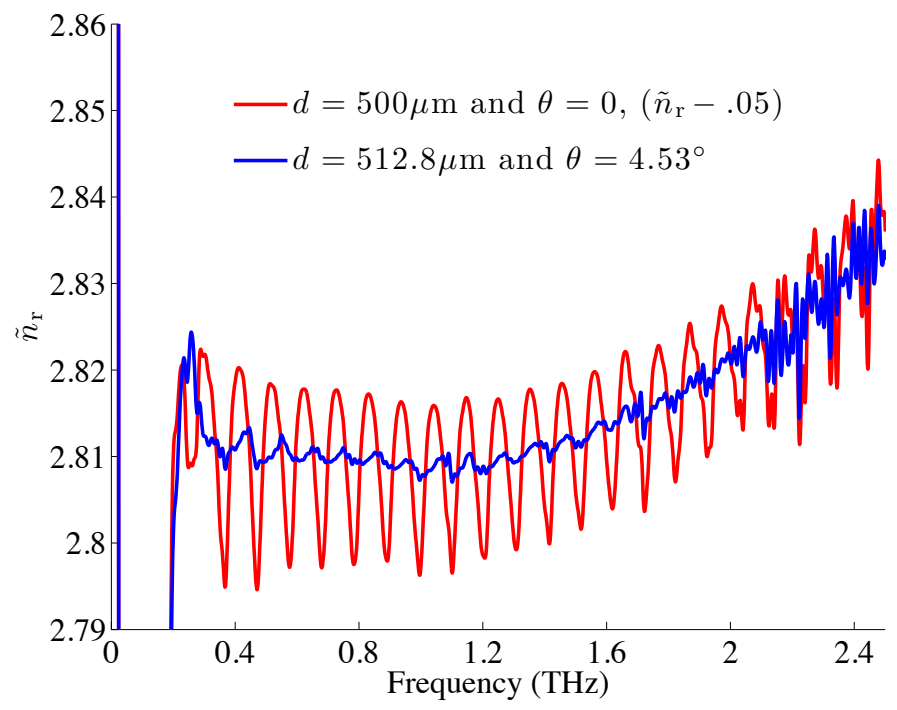

Fig. 7.5: Calculated Refractive index using assumed thickness of $0.5 \mathrm{~mm}$ and normal incident angle vs. final estimate of $512.8 \mu \mathrm{m}$ at a $4.53^{\circ}$ incidence.

46 , indicates the presence of a transverse optical phonon frequency at $12.25 \mathrm{THz}$ when compared with the pseudo-harmonic phonon model. This is the $\mathrm{E}_{1}$-TO transverse optical phonon frequency at about $12.25 \mathrm{THz}[22]$ as determined from Raman and Infrared spectroscopic techniques. This is the expected phonon resonance to be observed with the $\mathrm{THz}$ pulse propagating along the c-axis of the crystalline structure. At frequencies below $1.2 \mathrm{THz}$ the experimentally measured results diverge from the pseudo-harmonic phonon model, a feature observed in bulk ZnO THzTDS measurements as presented from other investigations [24]. The classic coupled plasmon-phonon model in eq. (6.2) on page 33 fails to predict the observed response, as did the inclusion of a Debye relaxation term. Only the modified Drude model that includes the plasma damping factor coupled with the phonon model predicted the observed dielectric response over the full span of reliable results.

Discrepancies in the experimental results with the pseudo-harmonic phonon 
model are even more pronounced in the imaginary component of the dielectric response $\varepsilon_{\mathrm{i}}$, as seen in Figure 7.7 on page 46 . Not only does the modified Drude model resolve the considerable offset between $\alpha$ and the phonon model, as seen in Figure 7.10, it models the curvature in the measured response observed in the imaginary component of the dielectric and refractive index as seen in Figures 7.7 and 7.9.

A previously reported [4] high frequency dielectric constant of 3.705 was used to model the bulk response in this work. A static dielectric constant of 7.82 in the modified coupled plasmon-phonon model and 7.84 in the pseudo-harmonic phonon model was found to best match the measurements, both of which fall in the range of reported values [11]. The phonon damping constant $\Gamma$, a measure of the full width at half maximum (FWHM) of the transverse optical phonon peak, was found to be about $0.5 \mathrm{THz}$, which is less than $0.82 \mathrm{THz}$ reported in another study [20].

The free carrier concentration was found to be less than $3 \times 10^{13} \mathrm{~cm}^{-3}$. The impact of slightly higher carrier concentrations than $3 \times 10^{13} \mathrm{~cm}^{-3}$ in the free carrier model is an increase in the extinction coefficient at lower frequencies and non-zero DC conductivities. Beyond $10^{14} \mathrm{~cm}^{-3}$ the model significantly diverges from the experimental results. The only reported carrier concentration for bulk crystalline $\mathrm{ZnO}$ grown with the hydrothermal method is $8 \times 10^{13} \mathrm{~cm}^{-3}$ [13]. The local maximum in the measured extinction coefficient, observed in Figure 7.9 on page 47 is related to the carrier damping constant in the presence of the plasma damping constant. A free carrier damping constant of $0.98 \mathrm{THz}$ was found to agree well with the experimental results when coupled with a plasma damping constant of $1.07 \mathrm{THz}$. The impact of the plasma damping constant is to resolve the discrepancy between the pseudoharmonic phonon model and the measured real component of the refractive index below 1.2 THz. Greater plasma damping constants model steeper divergent slopes 


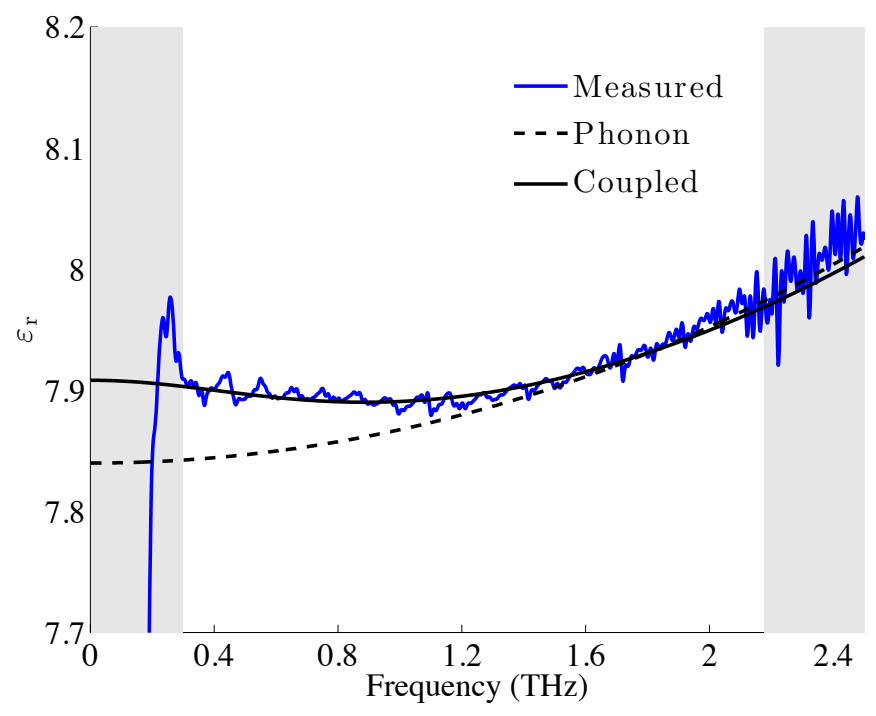

Fig. 7.6: The extracted real dielectric response from bulk crystalline $\mathrm{ZnO}$ as compared to the pseudo-harmonic phonon model (dashed, eq. (6.3)), and the modified Drude coupled plasmon-phonon model (solid, eq. (6.8)).

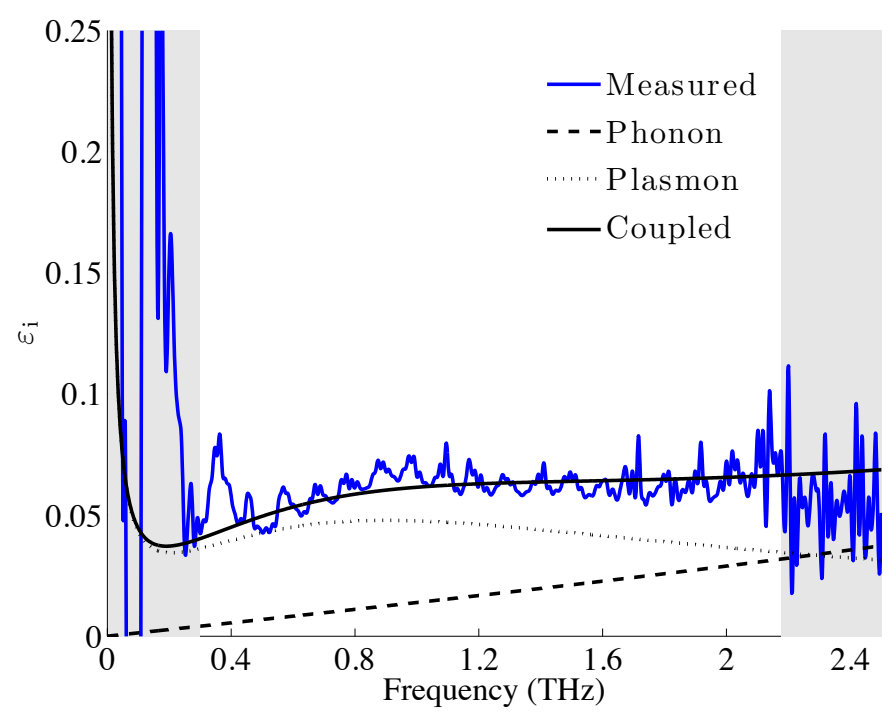

Fig. 7.7: The extracted imaginary dielectric response from bulk crystalline $\mathrm{ZnO}$ as compared to the pseudo-harmonic phonon model (dashed, eq. (6.3)), the plasmon response from the modified Drude model (dots, eq. (6.6)), and the modified Drude coupled plasmon-phonon model (solid, eq. (6.8)). 


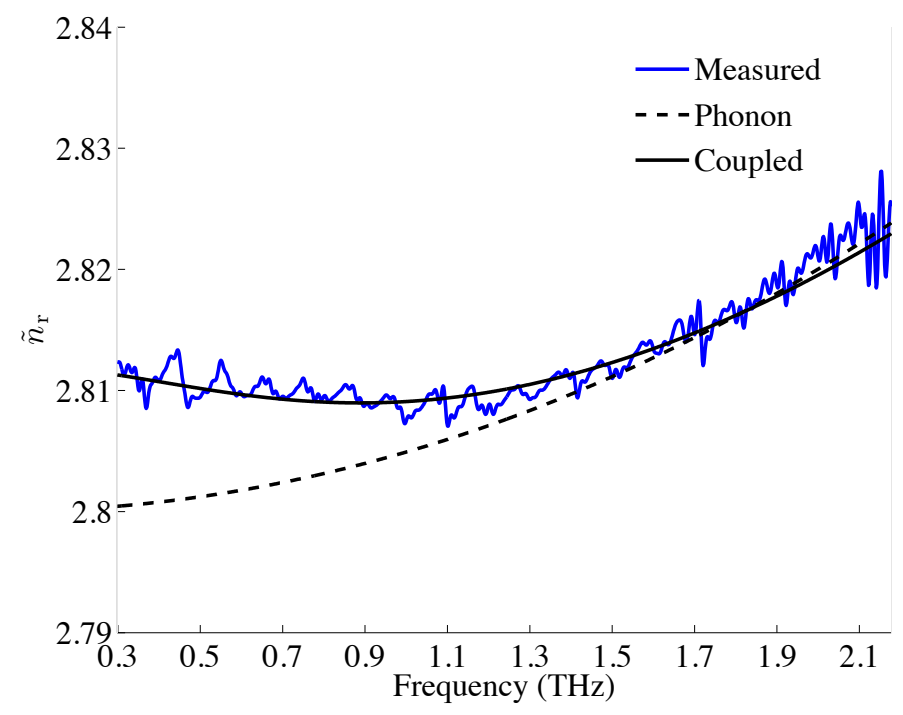

Fig. 7.8: Extracted $\mathrm{ZnO}$ wafer refractive index compared to the pseudo-harmonic phonon model (dashed, eq. (6.3)) and the improved coupled plasmon-phonon model (solid, eq. (6.8)).

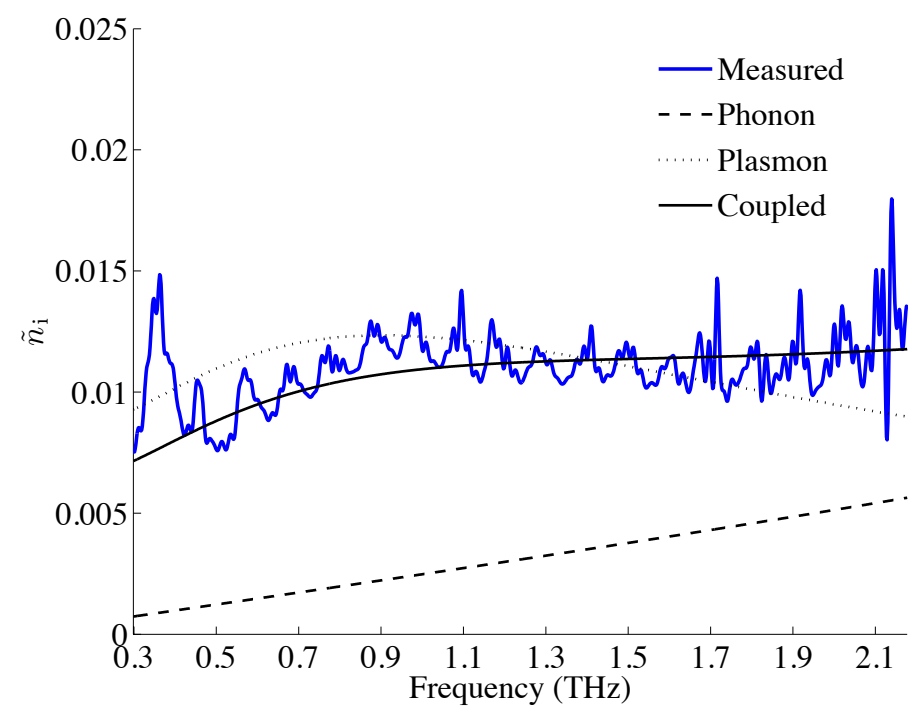

Fig. 7.9: Extracted $\mathrm{ZnO}$ wafer extinction coefficient compared to the pseudoharmonic phonon model (dashed, eq. (6.3)), the modified Drude model (dots, eq. (6.6)), and the modified coupled plasmon-phonon model (solid, eq. (6.8)). 


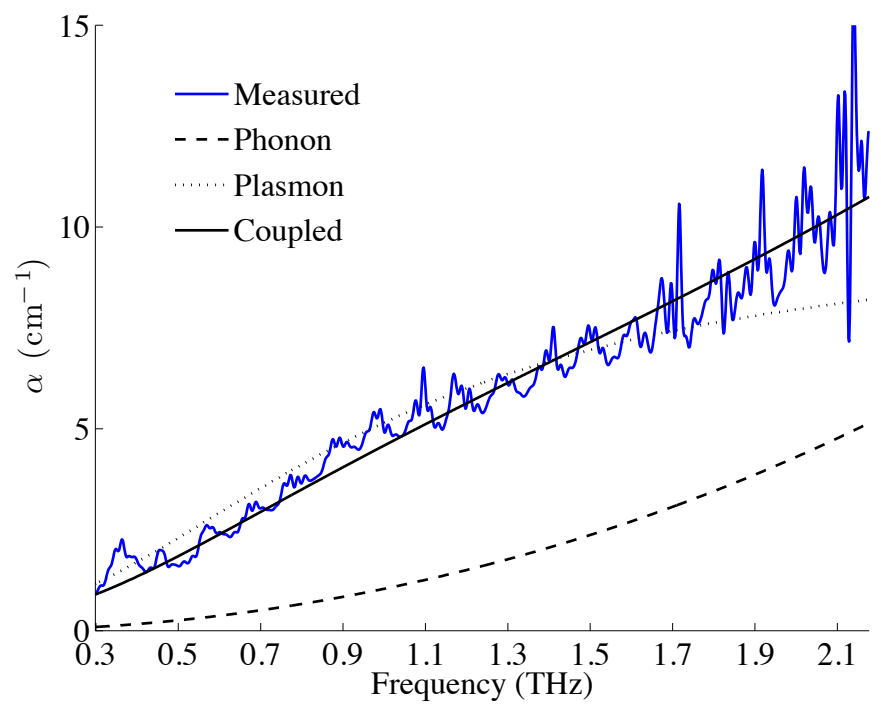

Fig. 7.10: Extracted $\mathrm{ZnO}$ wafer power absorption compared to the pseudo-harmonic phonon model (dashed, eq. (6.3)) and the modified coupled plasmon-phonon model (solid, eq. (6.8)).

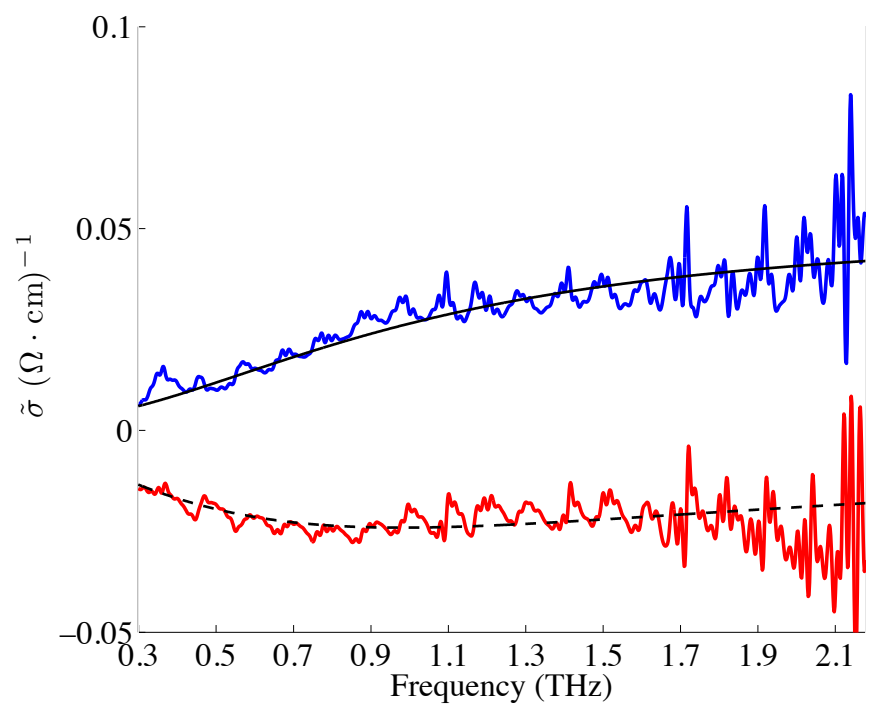

Fig. 7.11: Real (blue) and imaginary (red) components of the native conductive response as compared to the real component (solid) of the modified Drude model from eq. (6.14), and the imaginary component (dashed). 
Table 7.1: Model Parameters for Bulk Crystalline ZnO

\begin{tabular}{|c|c|c|c|c|c|c|}
\hline$\varepsilon(0)$ & $\varepsilon_{\infty}$ & $\begin{array}{c}\omega_{\mathrm{TO}} / 2 \pi \\
(\mathrm{THz})\end{array}$ & $\begin{array}{l}\Gamma / 2 \pi \\
(\mathrm{THz})\end{array}$ & $\begin{array}{l}\gamma_{0} / 2 \pi \\
(\mathrm{THz})\end{array}$ & $\begin{array}{l}\gamma_{\mathrm{pl}} / 2 \pi \\
(\mathrm{THz})\end{array}$ & $\begin{array}{c}N_{e} \\
\left(\mathrm{~cm}^{-3}\right)\end{array}$ \\
\hline \multicolumn{7}{|c|}{ (This work) } \\
\hline 7.82 & $3.705^{\#}$ & $12.25^{\#}$ & 0.5 & 0.98 & 1.07 & $\leq 3 \times 10^{13}$ \\
\hline \multicolumn{7}{|c|}{ (Previous report using THz-TDS [4]) } \\
\hline $7.77^{\#}$ & $3.705^{\#}$ & 12.42 & 0.82 & - & - & - \\
\hline \multicolumn{7}{|c|}{ (Parameters obtained from other methods) } \\
\hline $\begin{array}{l}7.61- \\
7.77[11]\end{array}$ & $\begin{array}{c}3.68- \\
3.705[11]\end{array}$ & $\begin{array}{c}12.19- \\
12.37[22]\end{array}$ & - & - & - & $8 \times 10^{13}[13]$ \\
\hline
\end{tabular}

away from the pseudo-harmonic phonon model at low frequencies and a larger nonzero offset in the extinction coefficient. The optimal plasma carrier constant was found to best model both the real and imaginary components of the experimentally measured complex refractive index.

In the non-photoexcited state intrinsic $\mathrm{ZnO}$ is expected to have a very low electrical conductivity. Of particular interest to this work, however, is if conductive differences can be determined between the bulk and nanowire morphologies. As indicated in section 6.2, in order to compare Drude model to experimental results it is necessary to remove the scattering and absorption due to optical phonons from the measurement. Even though this introduces some error it is useful for qualitative analysis. An extraction of the complex conductivity for bulk $\mathrm{ZnO}$ appears in Figure 7.11 on page 48 , from which can be seen a very low electrical conductivity of less than $0.05(\Omega \cdot \mathrm{cm})^{-1}$ out to about $2 \mathrm{THz}$.

A summary of the material parameters used to fit the models to the experimentally measured results appears in Table 7.1 above. 


\subsection{ZnO Nanowires}

Experiments were carried out at plate separations of $90 \mu \mathrm{m}, 201 \mu \mathrm{m}$, and $302 \mu \mathrm{m}$ in order to establish confidence in the effective medium method, the results of which can be found in Figure 7.14 on page 52. These plate separations were dependent on the available metal shims that had at least four shims of equal thickness, as determined from a vernier micrometer.

The general agreement in results below the respective $\mathrm{TM}_{1}$ cutoff for each plate spacing verified the usefulness of the effective medium model, and exposed an artifact that can be seen at $0.6 \mathrm{THz}$ in the $90 \mu \mathrm{m}$ results to be from waveguide coupling and not material response. Even though the chamber was purged with dry air during measurements some atmospheric effects remained. The multiple absorption dips above $1 \mathrm{THz}$, as seen in Figure 7.13 on page 51, are due to $\mathrm{H}_{2} \mathrm{O}$ vapor [32].

Using the ratio of average nanowire height to plate separation (1:90) in the effective medium model the refractive index of the $\mathrm{ZnO} /$ air composite was found to be approximately 1.8, as found in Figure 7.14 on page 52 . This value has been previously reported for a $\mathrm{ZnO} /$ air composite of annealed nanowires [5], which supports the result found in this work. Additionally, an investigation into the optical properties of spray pyrolysis prepared $\mathrm{ZnO}$ thin films by an ultraviolet-visible-near infrared spectrophotometer (UV-VIS-NIR) revealed the refractive index to be 1.8 in the near infrared region [33].

Consistent with the bulk crystalline results obtained from free space THz-TDS, the nanowire results obtained from the PPWG method as seen in Figures 7.15 and 7.16 on page 54 also exhibit a plasma damping behavior below $1 \mathrm{THz}$. This correlation supports the findings from the PPWG method and strengthens the validity of the MD-CPP model. 


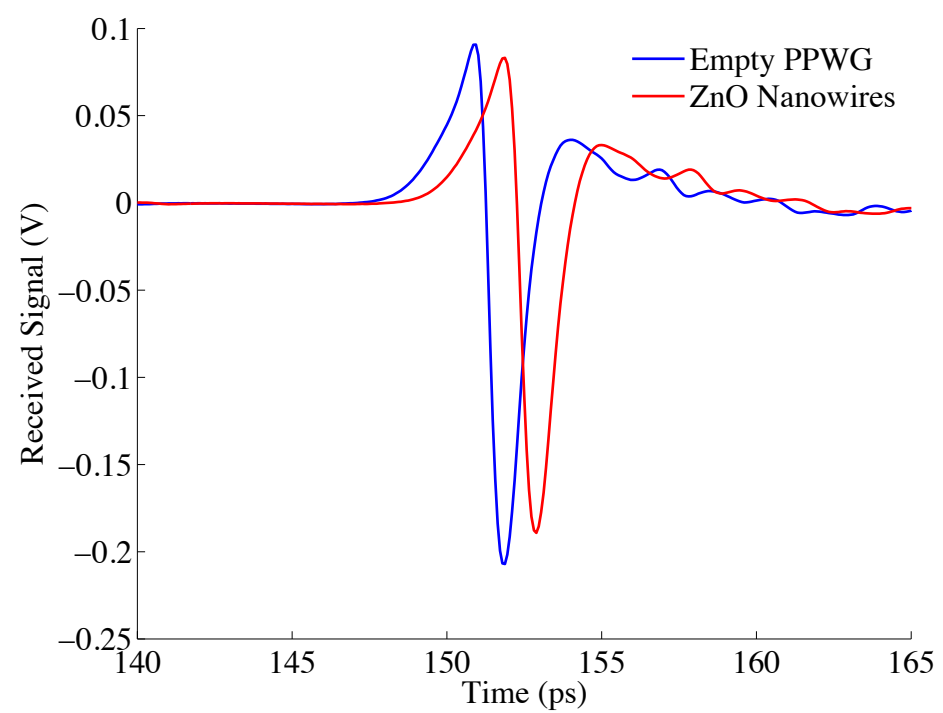

Fig. 7.12: Experimentally measured time domain response.

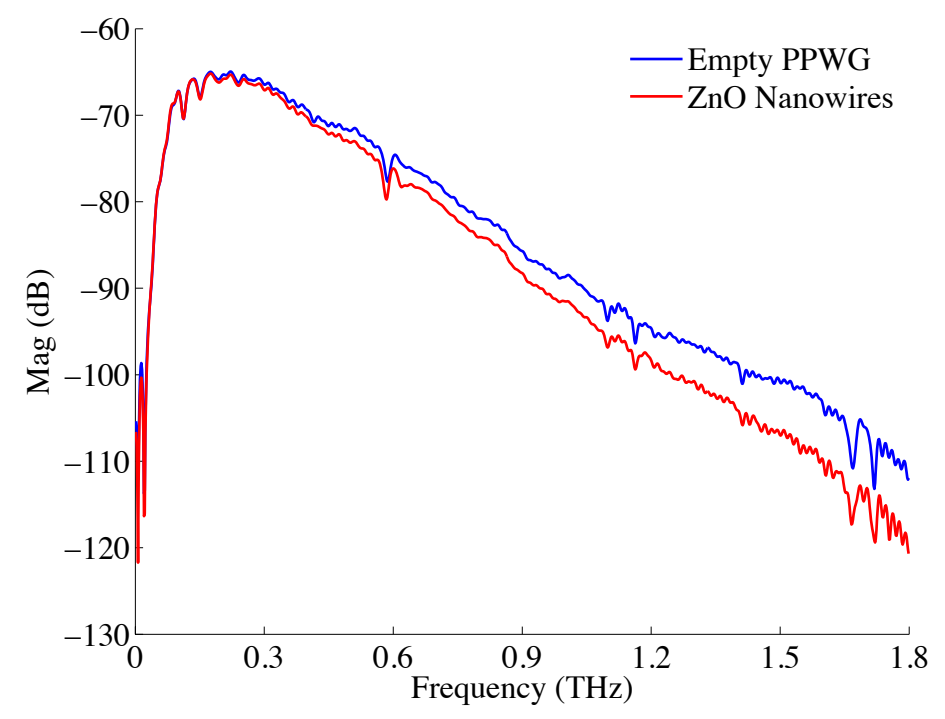

Fig. 7.13: Experimentally measured frequency domain response. 


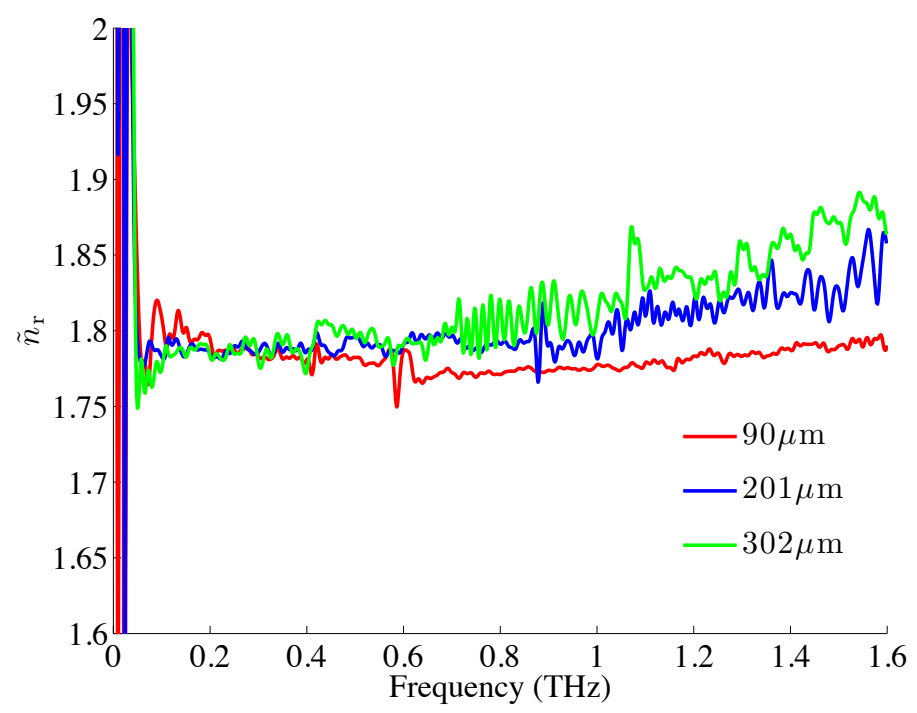

Fig. 7.14: Comparison of results extracted using the effective medium model for three different plate separations.

When these results were compared to the theoretic models the material parameter fit indicated the lowest transverse optical phonon resonance to be much lower than the expected $\mathrm{E}_{2}$-high mode at $13.09 \mathrm{THz}[22]$, consistent with what would be excited with the $\mathrm{THz}$ pulse propagating normal to the c-axis of $\mathrm{ZnO}$ wurtzitic structure. The response observed in this work near $6.14 \mathrm{THz}\left(209 \mathrm{~cm}^{-1}\right)$ has been previously observed in $\mathrm{ZnO}$ nanoparticles and attributed to the second order zone boundary (M-point) phonon 2-TA(M) [34]. The same measurement that revealed a 6.14 THz response also had a strong $\mathrm{E}_{2}$-high resonance at $13.09 \mathrm{THz}$, which indicated the orientation and crystalline structure was consistent with what has been found with confocal Raman spectroscopy performed on a single $\mathrm{ZnO}$ nanowire [35].

To best compare material parameters and the native conductivity between bulk crystalline and nanowire forms an estimation of $\mathrm{ZnO}$ density within the height of the $\mathrm{ZnO} /$ air composite layer was made to match the refractive index of the bulk 
material. A nanowire density of about $31.5 \%$ was found to return the expected bulk $\mathrm{ZnO}$ refractive index, which can be found in Figure 7.17 on page 55. At this new fill ratio the curvature of the refractive index still points to a transverse optical phonon frequency at about $6.14 \mathrm{THz}$ with a damping constant of $2 \mathrm{THz}$, as indicated by how $\Gamma$ provided a fit to the extinction coefficient in Figure 7.18. This implied that transverse optical phonon parameters are not subject to fill factor uncertainty in the effective medium model. The free carrier damping constant $\gamma_{0}$ still appeared to be $0.5 \mathrm{THz}$, and no change could be observed in the free carrier concentration. As expected, the extracted absorption for the pure $\mathrm{ZnO}$ nanowires in Figure 7.19 on page 56 was greater than that of the composite layer. The material parameters that did change were the static and high frequency dielectric constants from 3.11 and 1.75 to 7.7 and 3.5 respectively, and the plasma damping constant from $0.56 \mathrm{THz}$ to $0.65 \mathrm{THz}$. The parameters used in this work as compared to those previously reported appear in Table 7.2 on page 57 .

The observation of negative imaginary conductivity along with near zero DC conductivity in Figure 7.20 on page 56 has been previously reported for some nanomaterials such as $\mathrm{ZnO}$ and $\mathrm{TiO}_{2}$ [36]. The Drude model modified by Smith [31] succeeds in predicting the behavior of some liquid metals and quasicrystals and has been applied to the study of various nanomaterial in other work [30].

If the phonon contribution to the dielectric response is ignored when extracting complex conductivity from the experimentally measured dielectric response, the imaginary conductivity will be significantly more negative. The Drude-Smith model can provide a reasonably good fit to results extracted in this manner, which indicates that if parameter extraction is not done carefully, the Drude-Smith model can inadvertently be used to model the combined plasmon and phonon scattering 


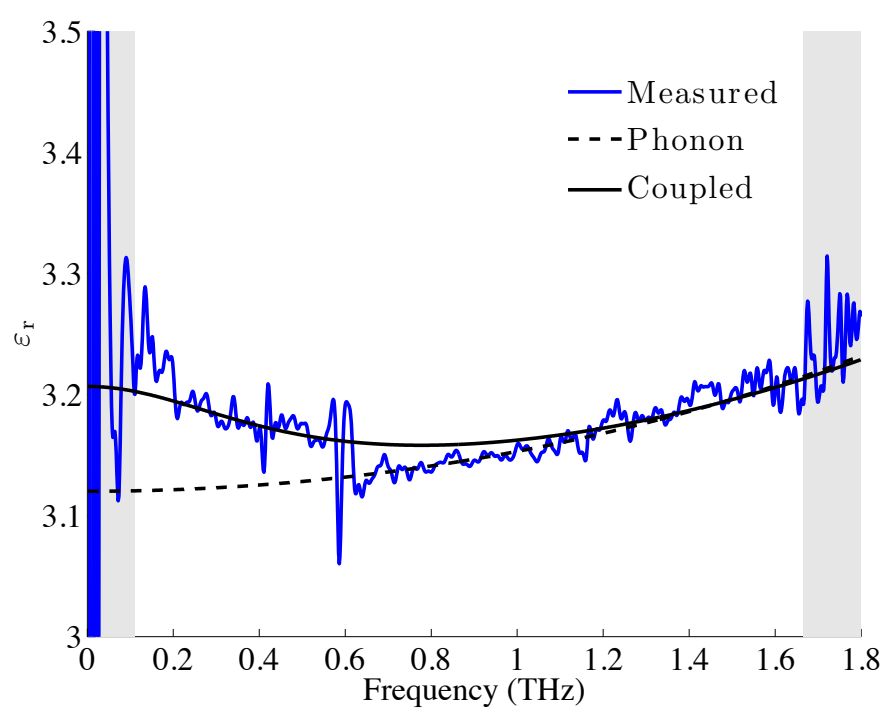

Fig. 7.15: The extracted real dielectric response from $\mathrm{ZnO}$ nanowires as compared to the pseudo-harmonic phonon model (dashed, eq. (6.3)), and the modified Drude coupled plasmon-phonon model (solid, eq. (6.8)).

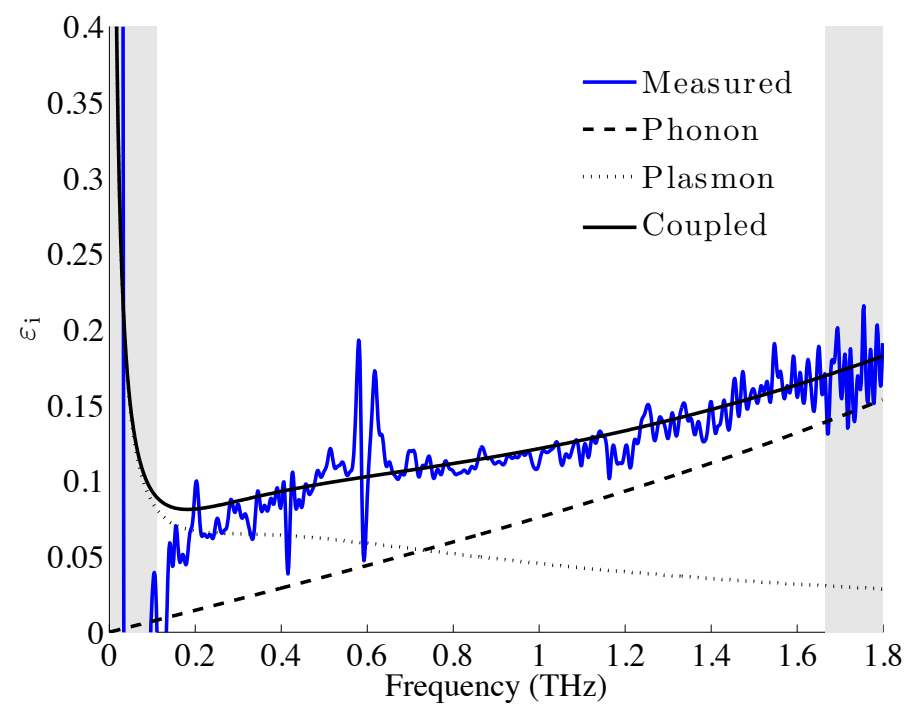

Fig. 7.16: The extracted imaginary dielectric response from $\mathrm{ZnO}$ nanowires as compared to the pseudo-harmonic phonon model (dashed, eq. (6.3)), the plasmon response from the modified Drude model (dots, eq. (6.6)), and the modified Drude coupled plasmon-phonon model (solid, eq. (6.8)). 


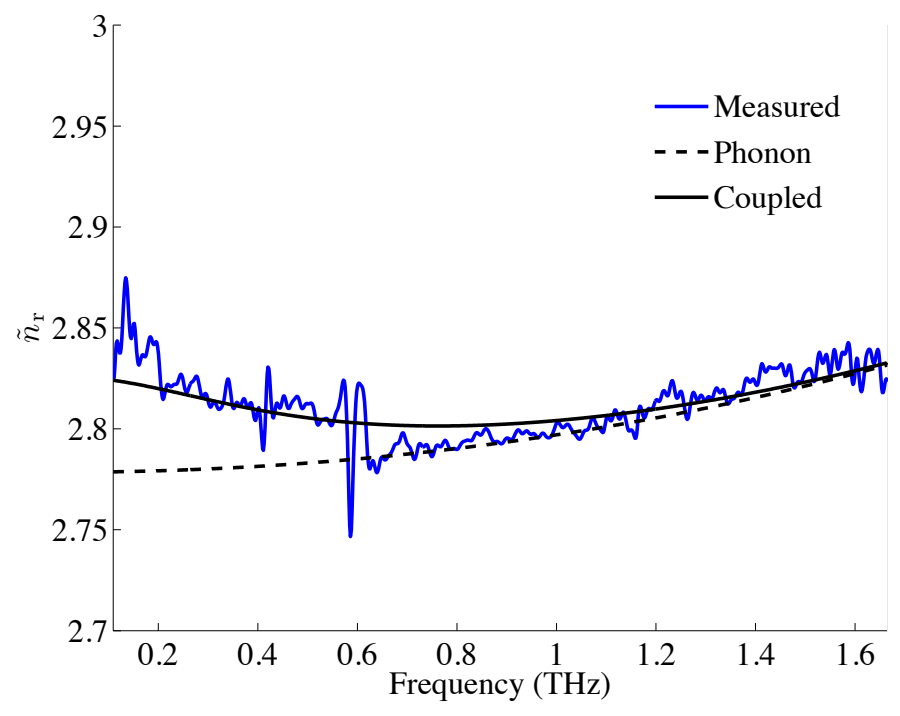

Fig. 7.17: Further extraction to yield the pure $\mathrm{ZnO}$ nanowire response from within the height of the $1 \mu \mathrm{m}$ tall $\mathrm{ZnO} /$ air composite layer.

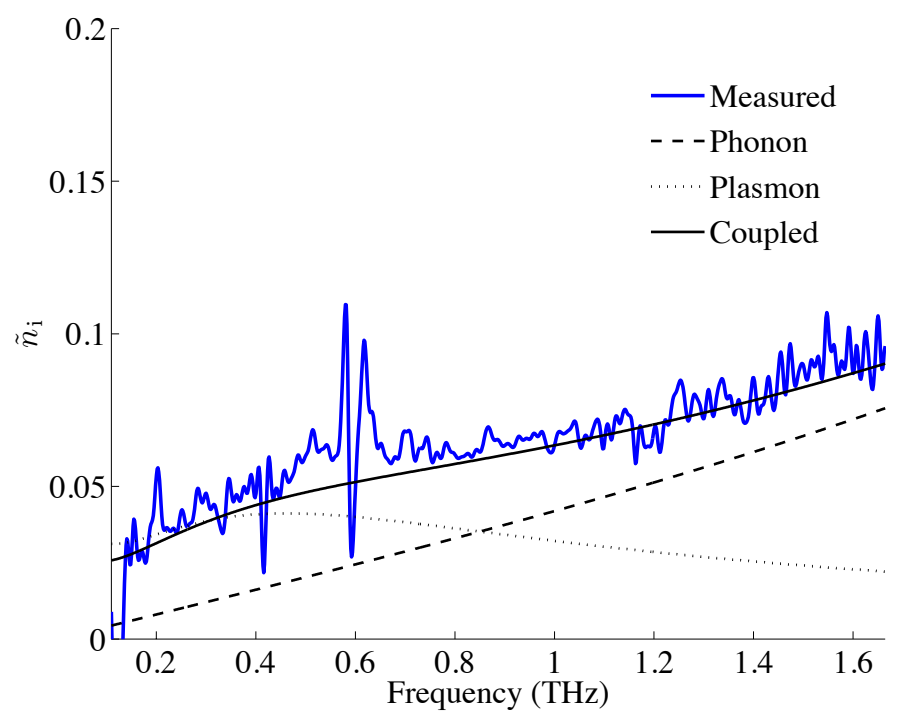

Fig. 7.18: The extracted extinction coefficient from pure $\mathrm{ZnO}$ nanowires as compared to the pseudo-harmonic phonon model (dashed, eq. (6.3)), the modified Drude model (dots, eq. (6.6)), and the modified coupled plasmon-phonon model (solid, eq. $(6.8))$. 


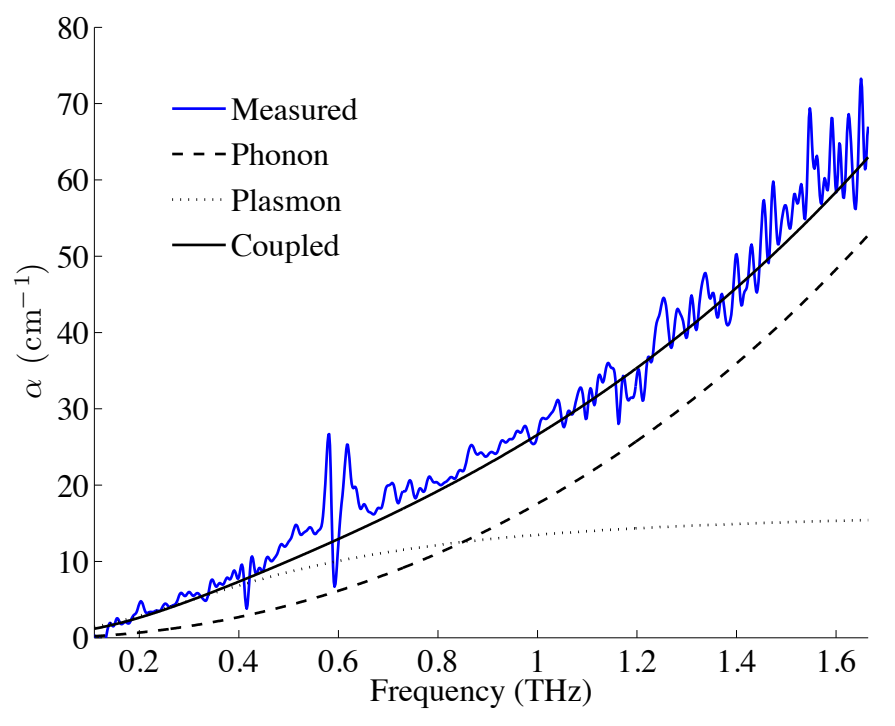

Fig. 7.19: The extracted power absorption from pure $\mathrm{ZnO}$ nanowires as compared to the pseudo-harmonic phonon model (dashed, eq. (6.3)), the modified Drude model (dots, eq. (6.6)), and the modified coupled plasmon-phonon model (solid, eq. (6.8)).

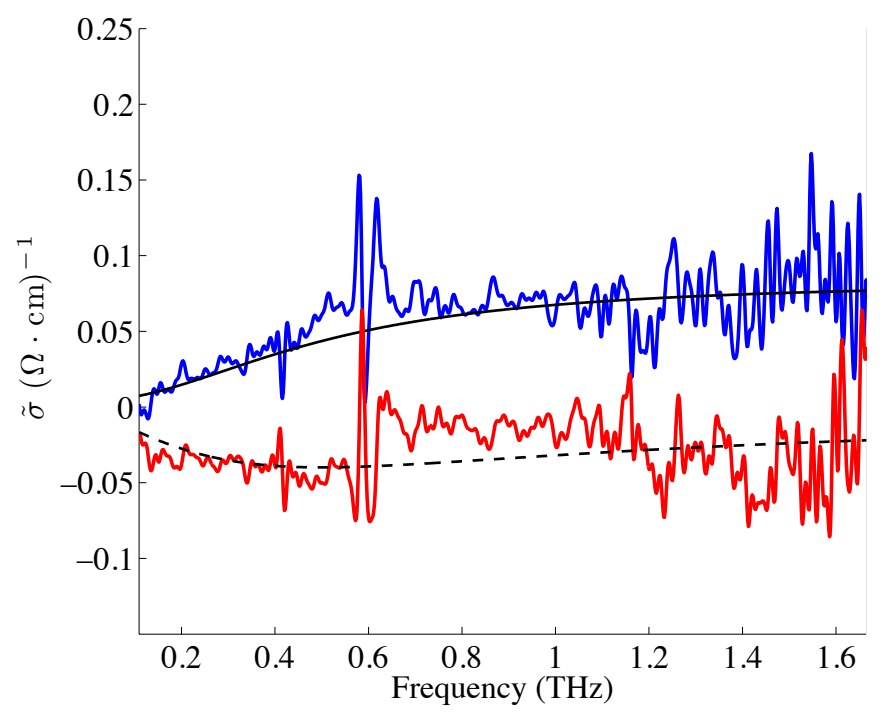

Fig. 7.20: Real (blue) and imaginary (red) components of the native conductive response as compared to the real component (solid) of the modified Drude model from eq. (6.14), and the imaginary component (dashed). 
Table 7.2: Model Parameters for ZnO Nanowires

\begin{tabular}{|c|c|c|c|c|c|c|c|}
\hline$f$ & $\varepsilon(0)$ & $\varepsilon_{\infty}$ & $\begin{array}{c}\omega_{\mathrm{TO}} / 2 \pi \\
(\mathrm{THz})\end{array}$ & $\begin{array}{l}\Gamma / 2 \pi \\
(\mathrm{THz})\end{array}$ & $\begin{array}{l}\gamma_{0} / 2 \pi \\
(\mathrm{THz})\end{array}$ & $\begin{array}{l}\gamma_{\mathrm{pl}} / 2 \pi \\
(\mathrm{THz})\end{array}$ & $\begin{array}{c}N_{e} \\
\left(\mathrm{~cm}^{-3}\right)\end{array}$ \\
\hline \multicolumn{8}{|c|}{ (This work) } \\
\hline 0.0111 & 3.11 & $1.75^{\#}$ & 6.14 & 2 & 0.5 & 0.56 & $\leq 3 \times 10^{13}$ \\
\hline 0.0035 & $7.7^{\#}$ & 3.5 & 6.14 & 2 & 0.5 & 0.65 & $\leq 3 \times 10^{13}$ \\
\hline \multicolumn{8}{|c|}{ (Previous reports using THz-TDS [4]) } \\
\hline 0.082 & 3.17 & 1.75 & $12.41 \pm 0.2$ & $12.5 \pm 0.2$ & - & - & - \\
\hline $0.01736^{\dagger}$ & 4.9 & 1.5 & $12.41 \pm 0.2$ & $21 \pm 0.2$ & - & - & - \\
\hline
\end{tabular}

processes instead of the electrical conductivity. Even though negative imaginary conductivity is observed in bulk and nanowire results the Drude-Smith model was not necessary since the plasma damping modification to the Drude model was found to be sufficient. For the first time a model for conductivity that is derived from the dielectric function has been presented that can reproduce the observed conductive behavior in the $0.1 \mathrm{THz}$ to $1.6 \mathrm{THz}$ range.

\subsection{Material Comparison}

A comparison of the extracted electrical conductivity in Figure 7.21 on page 58 reveals the composite layer of $\mathrm{ZnO}$ nanowires/air to have about the same effective conductivity as bulk crystalline $\mathrm{ZnO}$. As indicated by the additional extraction from the surrounding air in the composite layer, the nanowire morphology appears to be more conductive.

The parameters used in the modified coupled plasmon-phonon model can be found in Table 7.3 on page 58. As can be seen in Figures 7.22 and 7.23 on page 59 the theoretic model reveals a weak nanowire response as compared to bulk. Others have confirmed the presence of an $\mathrm{E}_{2}$-high optical phonon frequency at $13.09 \mathrm{THz}$ 


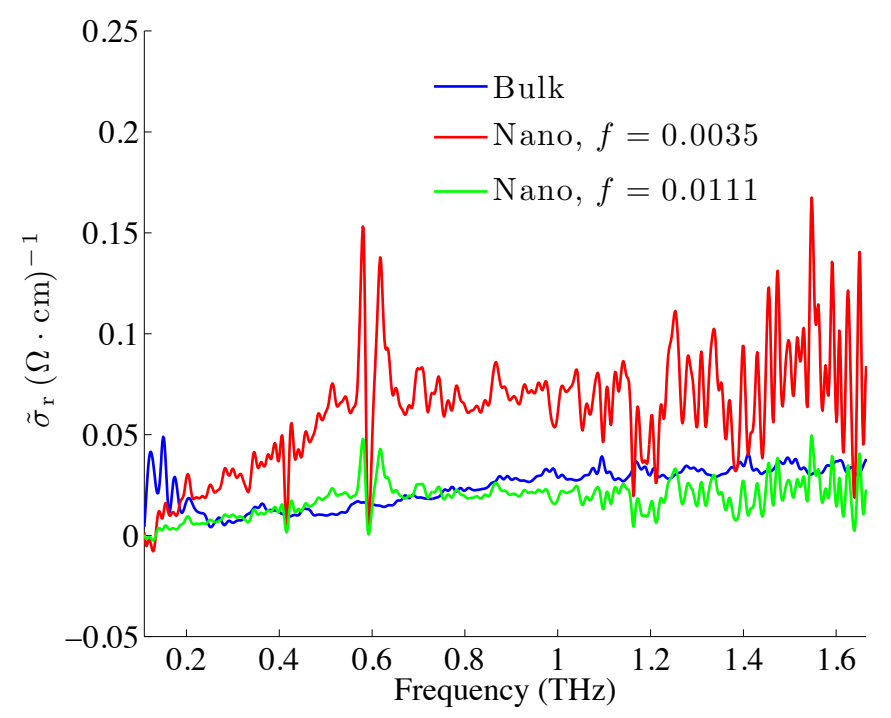

Fig. 7.21: A comparison of bulk vs. nanowire $\mathrm{ZnO}$ electrical conductivity.

$\left(413 \mathrm{~cm}^{-1}\right)$ in nanostructured $\mathrm{ZnO}$ [35] [34], but it may be that the detection of this mode is obscured by the presence of a lower phonon frequency not normally [22] present in the bulk material.

Table 7.3: Modeled Material Parameter Comparison

\begin{tabular}{|c|c|c|c|c|c|c|c|}
\hline$f$ & $\varepsilon(0)$ & $\varepsilon_{\infty}$ & $\begin{array}{c}\omega_{\mathrm{TO}} / 2 \pi \\
(\mathrm{THz}) \\
\end{array}$ & $\begin{array}{l}\Gamma / 2 \pi \\
(\mathrm{THz}) \\
\end{array}$ & $\begin{array}{l}\gamma_{0} / 2 \pi \\
(\mathrm{THz}) \\
\end{array}$ & $\begin{array}{l}\gamma_{\mathrm{pl}} / 2 \pi \\
(\mathrm{THz}) \\
\end{array}$ & $\begin{array}{c}N_{e} \\
\left(\mathrm{~cm}^{-3}\right) \\
\end{array}$ \\
\hline \multicolumn{8}{|c|}{ (Bulk Crystalline $\mathrm{ZnO}$ ) } \\
\hline 1 & 7.82 & $3.705^{\#}$ & $12.25^{\#}$ & 0.5 & 0.98 & 1.07 & $\leq 3 \times 10^{13}$ \\
\hline \multicolumn{8}{|c|}{ ZnO Nanowire/Air Composite } \\
\hline 0.0111 & 3.11 & $1.75^{\#}$ & 6.14 & 2 & 0.5 & 0.56 & $\leq 3 \times 10^{13}$ \\
\hline \multicolumn{8}{|c|}{ Pure $\mathrm{ZnO}$ Nanowires } \\
\hline 0.0035 & $7.7^{\#}$ & 3.5 & 6.14 & 2 & 0.5 & 0.65 & $\leq 3 \times 10^{13}$ \\
\hline
\end{tabular}




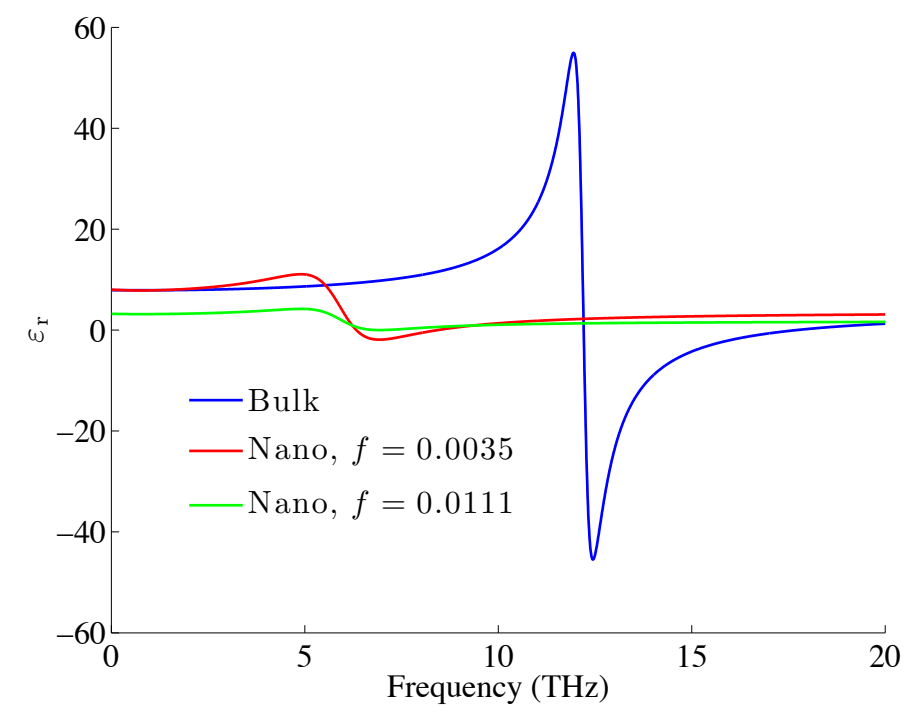

Fig. 7.22: Real component of the dielectric response based on theoretic parameter fit.

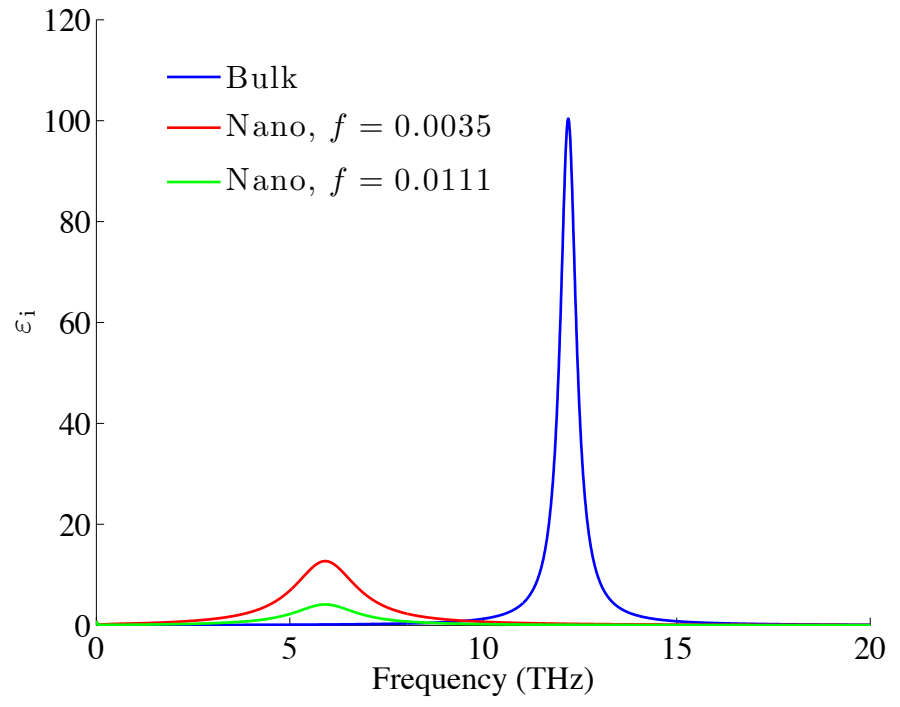

Fig. 7.23: Imaginary component of the dielectric response based on theoretic parameter fit. 


\section{Chapter 8}

\section{Conclusion}

The freespace material parameter extraction method was applied to a wafer of bulk crystalline $\mathrm{ZnO}$. The results agreed well when compared to previously reported results using THz-TDS. When compared to the pseudo-harmonic phonon model the bulk $\mathrm{ZnO}$ material parameters agreed well with established values for $\varepsilon(0), \varepsilon_{\infty}$, and $\omega_{\mathrm{TO}}$. The discrepancy between the pseudo-harmonic phonon model and the experimental results below $1 \mathrm{THz}$ was not unique to this investigation which led to the research that uncovered the modified Drude model. The exact physical process associated with this modification to the Drude model as it applies to THz-TDS is a subject for future work. Once the modified Drude model was incorporated into the coupled plasmon-phonon model the theoretic dielectric function agreed well with the experimental results over the full span of reliable measurements.

A method for applying non-differential THz-TDS techniques to nanostructures using a parallel-plate waveguide was developed and presented. ZnO nanowire growth on a waveguide plate was successful once the aluminum blocks were electroplated with gold, as confirmed with SEM images of in situ material. A refractive index of $\sim 1.8$ found from the simple effective medium model given the ratio of plate separation to nanowire height agreed with previously reported results obtained for $\mathrm{ZnO}$ thin films from NIR techniques and annealed $\mathrm{ZnO}$ nanowires from $\mathrm{THz}-\mathrm{TDS}$ 
techniques.

As observed in the bulk $\mathrm{ZnO}$ results the coupled plasmon-phonon model with the modified Drude mechanism best reproduced the experimentally measured dielectric response. The model was successful in identifying the $\mathrm{E}_{1}$-TO transverse optical phonon frequency at $12.25 \mathrm{THz}$.

For the nanowires the dielectric model placed $\omega_{\mathrm{TO}}$ lower than expected at 6.14 $\mathrm{THz}$, a result independent of fill factor from the effective medium model. The low phonon frequency observed in the $\mathrm{ZnO}$ nanowires needs to be confirmed with other methods in order to confirm that the result is not due to scattering or surface plasmon effects.

A method to extract native electrical conductivity from non-photoexcited material was presented and followed by a comparison between $\mathrm{ZnO}$ bulk and nanowire intrinsic conductivity. This method requires subtraction of the dielectric model of the phonon response from the total experimentally measured dielectric response in order to separate phonon from electron contribution. This method was able to successfully model real and imaginary conductivity in bulk crystalline and nanowire ZnO. Negative imaginary conductivity was observed experimentally and the modified Drude model was sufficiently accurate to model it. Results of this and related work have been published in [37]. 


\section{Chapter 9}

\section{Future Work}

The PPWG should be redesigned to replace shim spacing with a screw thread mechanism in order to allow continuously variable spacing. This modification would aid in an investigation into the nature of the waveguide coupling artifacts at $0.6 \mathrm{THz}$ observed in this work. Most notably, variable plate separation would allow one to dial in a spacing that places the cutoff frequency $f_{c}$ outside the measurable bandwidth. Alternatively, under $\mathrm{TE}_{1}$ mode operation variable plate separations could be utilized in order to support a moving window of single mode operation.

Thin films and other nanomaterial such as laser annealed $\mathrm{ZnO}$ nanorods [38] could be measured and cataloged using the PPWG technique. Experiments carried out at lower temperatures and, if possible, photoexcitation would allow for a more thorough investigation into defect related scattering processes in various nanomaterials.

Additional experimentation is required to validate the modified Drude model as applied to THz-TDS measurements. And finally, a study of Raman or IR spectroscopy as applied to as-grown nanowires is needed in order to confirm the presence of the $6.14 \mathrm{THz}$ phonon response observed in this work. 


\section{References}

[1] Mike Cooke, "Filling the THz gap", Semiconductor Today, vol. 2, no. 1, pp. 39-43, February 2007.

[2] Yun-Shik Lee, Principles of Terahertz Science and Technology, chapter 3, Springer Science+Business Media, 2009.

[3] Christopher D. Stoik, Nondestructive evaluation of aircraft composites using terahertz time domain spectroscopy, PhD thesis, Air Force Institute of Technology, Wright-Patterson Air Force Base, Ohio, December 2008.

[4] Abul K. Azad, Jiaguang Han, and Weili Zhang, "Terahertz dielectric properties of high-resistivity single-crystal ZnO", Applied Physics Letters, vol. 88, pp. 021103, 2006.

[5] Jason B. Baxter and Charles A. Schmuttenmaer, "Conductivity of ZnO Nanowires, Nanoparticles, and Thin Films Using Time-Resolved Terahertz Spectroscopy", J. Phys. Chem. B, vol. 110, pp. 25229-25239, 2006.

[6] Zhiping Jiang, Ming Li, and X.-C. Zhang, "Dielectric constant measurement of thin films by differential time-domain spectroscopy", Applied Physics Letters, vol. 76, no. 22, pp. 3221-3223, May 2000.

[7] Samuel P. Mickan, Kwang-Su Leeb, Toh-Ming Lub, Jesper Munchc, Derek Abbotta, and X.-C. Zhang, "Double modulated differential THz-TDS for thin film dielectric characterization", Microelectronics Journal, vol. 33, pp. 1033 1042, 2002.

[8] A. Higgins, F. Kernan, and B. Pejcinovic, "Multimode characterization of parallel plate waveguide", in Microwave Measurement Symposium (ARFTG). ARFTG, 2010, vol. 76 .

[9] A. Higgins, B. Pejcinovic, C. Cowen, and F. Kernan, "An investigation of parallel-plate waveguide terahertz radiation input coupling", in Defense Security and Sensing Conference. SPIE, April 2011.

[10] Anderson Janotti and Chris G Van de Walle, "Fundamentals of zinc oxide as a semiconductor", Reports on Progress in Physics, vol. 72, pp. 126501, 2009. 
[11] Ü. Özgür, Ya. I. Alivov, C. Liu, A. Teke, M. A. Reshchikov, S. Doğan, V. Avrutin, S.-J. Cho, and H. Morkoc, "A comprehensive review of $\mathrm{ZnO}$ materials and devices", Journal of Applied Physics, vol. 98, 2005.

[12] Youngchan Kim, Jaewook Ahn, Bog G. Kim, and Dae-Su Yee, "Terahertz birefringence in zinc oxide", Japanese Journal of Applied Physics, vol. 50, pp. 030203, March 2011.

[13] Katsumi Maeda, Mitsuru Sato, Ikuo Niikura, and Tsuguo Fukuda, "Growth of 2 inch zno bulk single crystal by the hydrothermal method", Semiconductor Science and Technology, vol. 20, no. 4, pp. S49-S54, April 2005.

[14] R Könenkamp, R. C. Word, M. Dosmailov, J. Meiss, and A. Nadarajah, "Selective growth of single-crystalline $\mathrm{ZnO}$ nanowires on doped silicon", Journal of Applied Physics, vol. 102, pp. 056103, September 2007.

[15] Lionel Vayssieres, "Growth of Arrayed Nanorods and Nanowires of $\mathrm{ZnO}$ from Aqueous Solutions", Advanced Materials, vol. 5, pp. 464-466, 2003.

[16] B. O'Regan, D. T. Schwartz, S. M. Zakeeruddin, and M. Gratzel, "Electrodeposited nanocomposite $\mathrm{n} \pm \mathrm{p}$ heterojunctions for solid-state dye-sensitized photovoltaics", Advanced Materials, vol. 12, no. 17, pp. 1263-1267, September 2000 .

[17] Timothy D. Dorney, Richard G. Baraniuk, and Daniel M. Mittleman, "Material parameter estimation with terahertz time-domain spectroscopy", J. Opt. Soc. Am. A, vol. 18, no. 7, pp. 1562 - 1571, July 2001.

[18] Ioachim Pupeza, Rafal Wilk, and Martin Koch, "Highly accurate optical material parameter determination with $\mathrm{THz}$ time-domain spectroscopy", Optics Express, vol. 15, no. 7, pp. 4335 - 4350, April 2007.

[19] Michael Bass, Ed., Handbook of Optics, vol. IV, chapter 5, McGraw Hill, 3rd edition, 2010.

[20] Jiaguang Han, Abul K. Azad, and Weili Zhang, "Far-infrared characteristics of bulk and nanostructured wide-bandgap semiconductors", Journal of Nanoelectronics and Optoelectronics, vol. 2, pp. 222-233, 2007.

[21] M. Balkanski, Optical Properties of Solids, chapter 8, North Holland, 1972.

[22] H. Morkoç and Ü. Özgür, General Properties of ZnO, in Zinc Oxide: Fundamentals, Materials and Device Technology, chapter 1, Wiley-VCH Verlag GmbH \& Co. KGaA, 2009. 
[23] Yun-Shik Lee, Principles of Terahertz Science and Technology, chapter 5, Springer Science+Business Media, 2009.

[24] Jiaguang Han, Wei Chen, J. Zhang, Mingxia He, A. K. Azad, S. Ray, Y. Zhao, and Weili Zhang, "Terahertz Response of Bulk and Nanostructured ZnO", PIERS Online, vol. 4, no. 3, pp. 391-395, 2008.

[25] Jean-François Baumard and François Gervais, "Plasmon and polar optical phonons in reduced rutile $\mathrm{TiO}_{2-x}$ ", Physical Review B, vol. 15, no. 4, pp. 2316-2323, February 1977.

[26] M. Gruji-Brojin, M. Šepanovi, Z. Dohevi-Mitrovi, and Z. V. Popovi, "Infrared Study of Nonstoichiometric Anatase $\mathrm{TiO}_{2}$ Nanopowders", Science of Sintering, vol. 38, pp. 183-189, 2006.

[27] Han-Kwang Nienhuys and Villy Sundström, "Influence of plasmons on terahertz conductivity measurements", Applied Physics Letters, vol. 87, no. 1, pp. 012101, 2005 .

[28] Gabriel P. Kniffin and Lisa M. Zurk, "Model-Based Material Parameter Estimation for Terahertz Reflection Spectroscopy", IEEE Transactions on Terahertz Science and Technology, vol. 2, no. 2, pp. 231, March 2012.

[29] Jason B. Baxter and Charles A. Schmuttenmaer, "Carrier dynamics in bulk ZnO. I. Intrinsic conductivity measured by terahertz time-domain spectroscopy", Physical Review B, vol. 80, pp. 235205, December 2009.

[30] F. Hegmann, D. Cooke, and M. Walther, "The nature of terahertz conductivity in nanomaterials", in Optical Terahertz Society and Technology, March 2007.

[31] N. V. Smith, "Classical generalization of the Drude formula for the optical conductivity", Physical Review B, vol. 64, pp. 155106, September 2001.

[32] X. Xin, H. Altan, A. Saint, D. Matten, and R. R. Alfano, "Terahertz absorption spectrum of para and ortho water vapors at different humidities at room temperature", Journal of Applied Physics, vol. 100, pp. 094905, November 2006 .

[33] C. Gümüş, O.M. Ozkendir, H. Kavak, and Y. Ufuktepe, "Structural and optical properties of zinc oxide thin films prepared by spray pyrolysis method", Journal of Optoelectronics and Advanced Materials, vol. 8, no. 1, pp. 299 - 303, 2006.

[34] M. Rajalakshmi, Akhilesh K. Arora, B. S. Bendre, and Shailaja Mahamuni, "Optical phonon confinement in zinc oxide nanoparticles", Journal of Applied Physics, vol. 87, no. 5, pp. 2445, 2000. 
[35] Sheng Yun Wu, "Confocal raman mapping study of a single nanowire", Microscopy: Science, Technology, Applications and Education, 2010.

[36] E. Hendry, M. Koeberg, B. O'Regan, and M. Bonn, "Local Field Effects on Electron Transport in Nanostructured $\mathrm{TiO}_{2}$ Revealed by Terahertz Spectroscopy", Nano Letters, vol. 6, no. 4, pp. 755-759, 2006.

[37] F. Kernan, A. Nadarajah, J.A. Higgins, B. Pejcinovic, and R. Könenkamp, "Terahertz characterization of zinc oxide nanowires using parallel-plate waveguides", in Nanotechnology (IEEE-NANO), 2011 11th IEEE Conference on. IEEE-NANO, 2011, pp. 404-408.

[38] A Nadarajah and R Könenkamp, "Laser annealing of photoluminescent $\mathrm{ZnO}$ nanorods grown at low temperature", Nanotechnology, vol. 22, pp. 025205, December 2011. 
Appendix 


\section{Appendix A}

\section{Measurement Deviation}

To derive the measurement error affecting the extracted solution to the complex refractive index a model of the transfer function that includes additive white noise and statistical error must be used. Recall that eq. (5.1) on page 22 represents the deconvolved transfer function of the system being measured. The frequency dependent transfer function in real and imaginary components is

$$
H=\frac{a+j b}{c+j d}=\frac{a c+b d+j(b c-a d)}{c^{2}+d^{2}}
$$

with $a \doteq \Re\left\{\overline{E_{\text {samp }}^{e x}}(\omega)\right\}, b \doteq \Im\left\{\overline{E_{\text {samp }}^{e x}}(\omega)\right\}, c \doteq \Re\left\{\overline{E_{\text {ref }}^{e x}}(\omega)\right\}$, and $d \doteq \Im\left\{\overline{E_{\text {ref }}^{e x}}(\omega)\right\}$.

Intervals for white noise will be denoted as $\Delta_{N} x$, and frequency dependent statistical error as $\Delta_{S} x$, with $x \in\{a, b, c, d\}$. These intervals will then be used to determine the variation in the measured transfer function $H$ as $\Delta H$.

The frequency independent $\Delta_{N} x$ is found by first recording the covered detector with no signal present for the same number of averaged measurements as used for the sample and reference. Then, after isolating an array $N$ with $k$ points at the same indices as used in the windowed time-domain for the sample and reference measurements the mean is computed as follows:

$$
m_{x}=\frac{1}{k} \sum_{i=1}^{k} N_{i}
$$

The interval due to white noise is the standard deviation of the noise array,

$$
\Delta_{N} x=\sqrt{\frac{1}{k-1} \sum_{i=1}^{k}\left(N_{i}-m_{x}\right)^{2}} .
$$

We let $\Delta_{S} x$ be the frequency dependent confidence interval of $\mathrm{x}$ due to statistical

error over $u$ measurements taken. The arrays are denoted $x^{(1)}, x^{(2)}, \ldots, x^{(u)}$, and the 
mean $x^{(m e a n)}$ is computed as:

$$
x^{(\text {mean })}=\frac{1}{u} \sum_{i=1}^{u} x^{(i)}
$$

The interval due to statistical error is the frequency dependent standard deviation of all measurements,

$$
\Delta_{S} x=\sqrt{\left.\frac{1}{u-1} \sum_{i=1}^{u}\left(x^{(i)}-x^{(\text {mean })}\right)\right)^{2}} .
$$

Since the two error sources are statistically independent, we find the total error interval to be

$$
\Delta x=\sqrt{\left(\Delta_{N} x\right)^{2}+\left(\Delta_{S} x\right)^{2}} .
$$

From equation (A.1) the real and imaginary components can be separated to get:

$$
\begin{aligned}
& \Re\{H\}=\frac{a c+b d}{c^{2}+d^{2}} \doteq f(a, b, c, d), \\
& \Im\{H\}=\frac{b c-a d}{c^{2}+d^{2}} \doteq g(a, b, c, d) .
\end{aligned}
$$

The Gaussian error propagation rule is then applied to yield:

$$
\begin{aligned}
& \Delta \Re\{H\}=\sqrt{\left(\Delta a \frac{\partial f}{\partial a}\right)^{2}+\left(\Delta b \frac{\partial f}{\partial b}\right)^{2}+\left(\Delta c \frac{\partial f}{\partial c}\right)^{2}+\left(\Delta d \frac{\partial f}{\partial d}\right)^{2}}, \\
& \Delta \Im\{H\}=\sqrt{\left(\Delta a \frac{\partial g}{\partial a}\right)^{2}+\left(\Delta b \frac{\partial g}{\partial b}\right)^{2}+\left(\Delta c \frac{\partial g}{\partial c}\right)^{2}+\left(\Delta d \frac{\partial g}{\partial d}\right)^{2}} .
\end{aligned}
$$

